\title{
Existence Results for Caputo-Hadamard Nonlocal Fractional Multi-Order Boundary Value Problems
}

\author{
Shahram Rezapour ${ }^{1,2}{ }^{(\mathbb{D}}$, Salim Ben Chikh ${ }^{3}$, Abdelkader Amara ${ }^{3}$, Sotiris K. Ntouyas ${ }^{4,5}{ }^{(D}$, Jessada Tariboon ${ }^{6, *(\mathbb{D})}$ \\ and Sina Etemad ${ }^{2}$ (D) \\ 1 Department of Medical Research, China Medical University Hospital, China Medical University, \\ Taichung 404, Taiwan; rezapourshahram@yahoo.ca \\ 2 Department of Mathematics, Azarbaijan Shahid Madani University, Tabriz 51368, Iran; \\ sina.etemad@gmail.com \\ 3 Laboratory of Applied Mathematics, University of Kasdi Merbah Ouargla, Ouargla 30000, Algeria; \\ benchikh.salim@univ-ouargla.dz (S.B.C.); amara.abdelkader@univ-ouargla.dz (A.A.) \\ 4 Department of Mathematics, University of Ioannina, 45110 Ioannina, Greece; sntouyas@uoi.gr \\ 5 Nonlinear Analysis and Applied Mathematics (NAAM)-Research Group, Department of Mathematices, \\ Faculty of Science, King Abdulaziz University, P.O. Box 80203, Jeddah 21589, Saudi Arabia \\ 6 Intelligent and Nonlinear Dynamic Innovations Research Center, Department of Mathematics, \\ Faculty of Applied Science, King Mongkut's University of Technology North Bangkok, \\ Bangkok 10800, Thailand \\ * Correspondence: jessada.t@sci.kmutnb.ac.th
}

check for updates

Citation: Rezapour, S.; Chikh, S.B.; Amara, A.; Ntouyas, S.N.; Tariboon, J.; Etemad, S. Existence Results for Caputo-Hadamard Nonlocal Fractional Multi-Order Boundary Value Problems. Mathematics 2021, 9, 719. https://doi.org/10.3390/ math9070719

Academic Editor: Christopher Goodrich

Received: 4 March 2021

Accepted: 23 March 2021

Published: 26 March 2021

Publisher's Note: MDPI stays neutral with regard to jurisdictional claims in published maps and institutional affiliations.

Copyright: (C) 2021 by the authors. Licensee MDPI, Basel, Switzerland. This article is an open access article distributed under the terms and conditions of the Creative Commons Attribution (CC BY) license (https:/ / creativecommons.org/licenses/by/ $4.0 /)$.

\begin{abstract}
In this paper, we studied the existence results for solutions of a new class of the fractional boundary value problem in the Caputo-Hadamard settings. Moreover, boundary conditions of this fractional problem were formulated as the mixed multi-order Hadamard integro-derivative conditions. To prove the main existence results, we applied two well-known techniques in the topological degree and fixed point theories. Finally, we provide two examples to show the compatibility of our theoretical findings.
\end{abstract}

Keywords: Caputo-Hadamard derivative; condensing function; topological degree theory; fractional boundary value problem (FBVP)

\section{Introduction}

An arbitrary order calculus is regarded as one of the most widely used subbranches of applied mathematics, which includes a vast range of applications in other sciences. Such a usefulness is because of the high compatibility of the formulas and fractional operators introduced in aforesaid theory. Different researchers have utilized several newly-introduced fractional operators in recent times to describe some dynamical aspects of different kinds of real processes in the world based on their fractional modelings. Examples on the applicability of arbitrary order operators in the modeling of natural processes can be observed in different published literature works, including [1-11]. On the contrary, since making new modelings with the aid of fractional operators obtains more accurate numerical findings than modeling by means of the standard integer order operators, numerous mathematicians have tried to provide different novel extensions of some existing standard fractional operators every day. Two fractional operators that we intend to apply in this manuscript are the Hadamard integration, along with the Caputo-Hadamard differentiation operators. We can even refer the readers to articles in which the existing modelings are studied with the help of both aforementioned operators. See, for example, [12-16]. 
In 2017, Ntouyas and Tariboon [17] turned to a new framework of the boundary value problem via multiple orders on fractional operators, as follows:

$$
\left\{\begin{array}{l}
\zeta^{\mathcal{R}} \mathfrak{D} \varsigma u(t)+(1-\zeta)^{\mathcal{R}} \mathfrak{D}^{\theta^{*}} u(t)=h(t, u(t)), \\
u(0)=0, \quad \sigma^{*} \mathcal{R}_{\mathcal{I}} q_{1}^{*} u(R)+\left(1-\sigma^{*}\right)^{\mathcal{R}} \mathcal{I}^{q_{2}^{*}} u(R)=\delta_{2}^{*},
\end{array}\right.
$$

in which boundary conditions are regarded as Riemann-Liouville bi-order integral conditions and $t \in[0, R]$ and $\varsigma \in(1,2)$. The authors proved the existence results via Sadovski's fixed point theorem. After this, Lei, Qixang, and Gang [18] established some theorems based on the existence and Hyers-Ulam stability of solutions for the proposed fractional two-term multi-order boundary value problem:

$$
\left\{\begin{array}{l}
p^{\mathcal{R}} \mathfrak{D}^{k^{*}} \mu(t)+{ }^{\mathcal{R}} \mathfrak{D}^{\theta^{*}} \mu(t)=h(t, \mu(t)), \\
\mu(0)=0, \quad \sigma^{* \mathcal{R}} \mathfrak{D}^{\gamma_{1}^{*}} \mu(R)+{ }^{\mathcal{R}} \mathcal{I}^{q_{2}^{*}} \mu(v)=\delta_{2}^{*}, \quad\left(k^{*} \in(1,2)\right) .
\end{array}\right.
$$

The boundary conditions considered by them are in a two-term framework of mixed three-point Riemann-Liouville boundary conditions. The authors proved the existence results with the help of an integral inequality and established the stability results for null boundary conditions [18].

At this moment, according to the available ideas in these articles, we intend to address a general extension of these works by formulating the following Caputo-Hadamard fractional boundary value problem via mixed multi-order integro-derivative conditions:

$$
\left\{\begin{array}{l}
\lambda^{\mathcal{C H}} \mathfrak{D}_{1^{+}}^{\varsigma} u(t)+{ }^{\mathcal{C H}} \mathfrak{D}_{1^{+}}^{\theta^{*}} u(t)=\hat{A}(t, u(t)), \\
u(1)=0 \\
\mu_{1}^{* \mathcal{C H}} \mathfrak{D}_{1^{+}}^{\gamma_{1}^{*}} u(M)+{ }^{\mathcal{C H}} \mathfrak{D}_{1^{+}}^{\gamma_{2}^{*}} u(\eta)=\delta_{1}, \\
\mu_{2}^{* \mathcal{H}} \mathcal{I}_{1^{+}}^{q_{1}^{*}} u(M)+{ }^{\mathcal{H}} \mathcal{I}_{1^{+}}^{q^{*}} u(\eta)=\delta_{2}
\end{array}\right.
$$

so that $\lambda, \mu_{1}^{*}, \mu_{2}^{*} \in(0,1], \gamma_{1}^{*}, \gamma_{2}^{*} \in\left(0, \varsigma-\theta^{*}\right)$ with $2<\theta^{*}<\varsigma<3, q_{1}^{*}, q_{2}^{*} \in \mathbb{R}^{+}, \delta_{1}, \delta_{2} \in \mathbb{R}$ and $t \in[1, M]$. The symbol $\mathcal{C H}_{\mathfrak{D}_{1+}^{\alpha^{*}}}$ points out the Caputo-Hadamard derivative of order $\alpha^{*} \in\left\{\varsigma, \gamma_{1}^{*}, \gamma_{2}^{*}, \theta^{*}\right\}$, with the notation $\mathcal{H}_{\mathcal{I}_{1+}^{q^{*}}}$ stands for the Hadamard integral of order $q^{*} \in\left\{q_{1}^{*}, q_{2}^{*}\right\}$. A map $\hat{A}$ formulated by $\hat{A}:[1, M] \times \mathbb{R} \rightarrow \mathbb{R}$ is assumed to be continuous on $[1, M] \times \mathbb{R}$ with respect to its both components. It is convenient that the researchers of this field notice this subject so that, motivated by the flexibility of boundary value problems (1) and (2), the multi-order Caputo-Hadamard-FBVP (3) is modeled with respect to the generalized operators with kernels, including logarithmic functions. In other words, the presented formulation for the given multi-order Caputo-Hadamard-FBVP (3) involves four different derivatives in the format of the Caputo-Hadamard, as well as two different integrals in the sense of Hadamard. This combined FBVP covers the previous standard cases of nonlinear fractional differential equations by assigning arbitrary values for all existing parameters and orders or defining the standard kernel in the mentioned FBVP (3). The supposed abstract fractional boundary value problem (3) with given mixed boundary conditions can describe some mathematical models of real and physical processes in which some parameters are often adjusted to suitable situations. The value of these parameters can change the effects of fractional derivatives and integrals. Moreover, we express that such a Caputo-Hadamard multi-order fractional problem has a new and general structure and is defined for the first time with the aid of the Caputo-Hadamard notion. In this way, we tried to find analytical existence criteria for the proposed problem (3) with the help of a composition of existing techniques in the topological degree and fixed point theories. For more details, see [19-22]. 
The scheme of the contents is as follows: First, several required contexts on fractional calculus and some notions about condensing operators are assembled. Section 3 is assigned to finding the criteria in which the existence properties can be derived by terms of the mixed techniques available in the topological degree and fixed point theories. In Section 4, our analytical findings are supported by demonstrating two simulation examples in the numerical setting to indicate the applicability of our proofs.

\section{Preliminaries}

First of all, some fundamental and auxiliary preliminaries on the arbitrary order calculus are assembled in this part of the manuscript. The $\varsigma^{\text {th }}$-Hadamard integral of a given function $u \in \mathcal{C}_{\mathbb{R}}([c, b])$ is formulated by:

$$
\mathcal{H}_{\mathcal{I}_{c^{+}}^{\varsigma}} u(t)=\frac{1}{\Gamma(\varsigma)} \int_{c}^{t}\left(\ln \frac{t}{r}\right)^{(\varsigma-1)} u(r) \frac{\mathrm{d} r}{r}
$$

when the right hand integral exists [13,23] and $\mathcal{H}_{\mathcal{I}_{c^{+}}^{0}} u(t)=u(t)$. Additionally, for each $\varsigma_{1}, \varsigma_{2} \in \mathbb{R}^{+}$, the semigroup property ${ }^{\mathcal{H}_{\mathcal{I}^{+}}^{\zeta_{1}}}\left({ }^{\mathcal{H}} \mathcal{I}_{c^{+}}^{\zeta_{2}} u(t)\right)={ }^{\mathcal{H}_{\mathcal{I}^{+}}}{ }_{c_{1}}^{\zeta_{1} \varsigma_{2}} u(t)$ settles and:

$$
\mathcal{H}_{\mathcal{I}_{c^{+}}^{\varsigma_{1}}}\left(\ln \frac{t}{c}\right)^{\varsigma_{2}}=\frac{\Gamma\left(\varsigma_{2}+1\right)}{\Gamma\left(\varsigma_{1}+\varsigma_{2}+1\right)}\left(\ln \frac{t}{c}\right)^{\varsigma_{1}+\varsigma_{2}}, \quad t>c .
$$

Taking $\varsigma_{2}=0$, it is clear that:

$$
\mathcal{H}_{\mathcal{I}_{c^{+}}^{\zeta_{1}} 1}=\frac{1}{\Gamma\left(\varsigma_{1}+1\right)}\left(\ln \frac{t}{c}\right)^{\varsigma_{1}} .
$$

In this position, we suppose that $n=[\varsigma]+1$. Then, the $\varsigma^{t h}$-Hadamard derivative for an arbitrary mapping $u:(c, b) \rightarrow \mathbb{R}$ is illustrated by:

$$
\mathcal{H}_{\mathfrak{D}_{c^{+}}^{\varsigma}} u(t)=\frac{1}{\Gamma(n-\varsigma)}\left(t \frac{\mathrm{d}}{\mathrm{d} t}\right)^{n} \int_{c}^{t}\left(\ln \frac{t}{r}\right)^{(n-\varsigma-1)} u(r) \frac{\mathrm{d} r}{r},
$$

if RHS exists $[13,23]$. In the following, the $\varsigma^{\text {th }}$-Caputo-Hadamard derivative for $u \in \mathcal{A C}_{\mathbb{R}}^{n}([c, b])$ is introduced by

$$
\mathcal{C H}_{\mathfrak{D}_{c^{+}}^{\varsigma}} u(t)=\frac{1}{\Gamma(n-\varsigma)} \int_{\mathcal{c}}^{t}\left(\ln \frac{t}{r}\right)^{(n-\varsigma-1)}\left(t \frac{\mathrm{d}}{\mathrm{d} t}\right)^{n} u(r) \frac{\mathrm{d} r}{r}
$$

if the existing integral has finite value $[13,23,24]$. Again, by assuming $u \in \mathcal{A C}_{\mathbb{R}}^{n}([c, b])$ and $n-1<\varsigma<n$ and in light of [23], it is confirmed that the series solution of the given homogeneous Caputo-Hadamard-FDE $\mathcal{C H}_{\mathfrak{D}_{c^{+}}}^{s} u(t)=0$ possesses a series structure as: $u(t)=\sum_{l=0}^{n-1} b_{l}\left(\ln \frac{t}{c}\right)^{l}$ and thus the following equality is valid for each $t>c$ :

$$
\mathcal{H}_{\mathcal{I}_{c^{+}}^{\varsigma}}\left(\mathcal{C H}_{\mathfrak{D}_{c^{+}}^{\varsigma}} u(t)\right)=u(t)+b_{0}+b_{1}\left(\ln \frac{t}{c}\right)+b_{2}\left(\ln \frac{t}{c}\right)^{2}+\cdots+b_{n-1}\left(\ln \frac{t}{c}\right)^{n-1} .
$$

We now regard $\mathbb{B}^{*}$ as the category of all bounded sets belonging to the Banach space $\mathcal{W}$. The measure of noncompactness attributed to Kuratowski (KMNC) $\mu: \mathbb{B}^{*} \rightarrow \mathbb{R}^{+}$is constructed by:

$$
\mu\left(\mathfrak{G}^{*}\right):=\inf \left\{\varepsilon>0: \mathfrak{G}^{*}=\cup_{l=1}^{n} \mathfrak{G}_{l}^{*} \text { and } \mathrm{D}\left(\mathfrak{G}_{l}^{*}\right) \leq \varepsilon, \quad l \in \mathbb{N}_{1}^{n}\right\},
$$

so that $\mathrm{D}\left(\mathfrak{G}_{l}^{*}\right)=\sup \left\{\left|u-u^{\prime}\right|: u, u^{\prime} \in \mathfrak{G}_{l}^{*}\right\}$ and $\mathfrak{G}^{*}$ stands for a bounded set contained in $\mathbb{B}^{*}$ and $\mu\left(\mathfrak{G}^{*}\right) \leq \mathrm{D}\left(\mathfrak{G}^{*}\right) \in(0, \infty)$. The symbol D points to the diameter here [25-27].

Lemma $1([25,28])$. Let $\mathfrak{G}^{*}, \mathfrak{O}^{*} \in \mathbb{B}^{*}$ be contained in $\mathcal{W}$, which is bounded. Then the following seven statements hold: 
(1) $\mu\left(\mathfrak{G}^{*}\right)=0$ if and only if $\mathfrak{G}^{*}$ is relatively compact;

(2) $\mu\left(\mathfrak{G}^{*}\right)=\mu\left(\overline{\mathfrak{G}^{*}}\right)=\mu\left(\operatorname{cnvx}\left(\mathfrak{G}^{*}\right)\right)$, in which $\operatorname{cnvx}\left(\mathfrak{G}^{*}\right)$ and $\overline{\mathfrak{G}^{*}}$ display the convex hull and the closure of $\mathfrak{G}^{*}$;

(3) $\mathfrak{G}^{*} \subseteq \mathfrak{O}^{*}$ gives $\mu\left(\mathfrak{G}^{*}\right) \leq \mu\left(\mathfrak{O}^{*}\right)$;

(4) $\mu\left(\sigma+\mathfrak{O}^{*}\right) \leq \mu\left(\mathfrak{O}^{*}\right)$ for any $\sigma \in \mathbb{R}$;

(5) $\mu\left(\sigma \mathfrak{G}^{*}\right)=|\sigma| \mu\left(\mathfrak{G}^{*}\right)$ for any $\sigma \in \mathbb{R}$;

(6) $\mu\left(\mathfrak{G}^{*}+\mathfrak{D}^{*}\right) \leq \mu\left(\mathfrak{G}^{*}\right)+\mu\left(\mathfrak{O}^{*}\right)$ subject to $\mathfrak{G}^{*}+\mathfrak{O}^{*}=\left\{u+u^{\prime} ; u \in \mathfrak{G}^{*}, u^{\prime} \in \mathfrak{O}^{*}\right\}$;

(7) $\mu\left(\mathfrak{G}^{*} \cup \mathfrak{O}^{*}\right) \leq \max \left\{\mu\left(\mathfrak{G}^{*}\right), \mu\left(\mathfrak{O}^{*}\right)\right\}$.

Let us take $\mathfrak{G}^{*} \in \mathbb{B}^{*}$ as a bounded subset contained in $\mathcal{W}$. A continuous bounded mapping $\Psi^{*}: \mathfrak{G}^{*} \rightarrow \mathcal{W}$ is termed to be $\mu$-Lipschitz whenever $\tilde{R}^{*} \geq 0$ exists so that:

$$
\mu\left(\Psi^{*}\left(\mathfrak{G}^{*}\right)\right) \leq \tilde{R}^{*} \mu\left(\mathfrak{G}^{*}\right) .
$$

In addition, we call $\Psi^{*}$ a strict $\mu$-contraction when $\tilde{R}^{*}<1$ [25]. A $\mu$-condensing mapping $\Psi^{*}$ is formulated by inequality $\mu\left(\Psi^{*}\left(\mathfrak{G}^{*}\right)\right) \leq \mu\left(\mathfrak{G}^{*}\right)$ for any $\mathfrak{G}^{*} \in \mathbb{B}^{*}$ via $\mu\left(\mathfrak{G}^{*}\right)>0$. From $\mu\left(\Psi^{*}\left(\mathfrak{G}^{*}\right)\right) \geq \mu\left(\mathfrak{G}^{*}\right)$ it follows that $\mu\left(\mathfrak{G}^{*}\right)=0[25,29,30]$.

Proposition 1 ([31]). Suppose that $\Psi^{*}: \mathfrak{G}^{*} \subset \mathcal{W} \rightarrow \mathcal{W}$ is Lipschitz with constant $\tilde{R}^{*}$. Then, $\Psi^{*}$ is $\mu$-Lipschitz with constant $\tilde{R}^{*}$.

Proposition 2 ([31]). Let $\Psi^{*}: \mathfrak{G}^{*} \subset \mathcal{W} \rightarrow \mathcal{W}$ be compact for any $\mathfrak{G}^{*} \subset \mathcal{W}$. Then, $\Psi^{*}$ is $\mu$-Lipschitz via $\tilde{R}^{*}=0$.

Proposition 3 ([31]). For any $\mathfrak{G}^{*} \subset \mathcal{W}$, both operators $\Psi_{1}^{*}, \Psi_{2}^{*}: \mathfrak{G}^{*} \rightarrow \mathcal{W}$ are supposed to be $\mu$-Lipschitz with constants $\tilde{R}_{1}^{*}$ and $\tilde{R}_{2}^{*}$. In this case, $\Psi_{1}^{*}+\Psi_{2}^{*}: \mathfrak{G}^{*} \rightarrow \mathcal{W}$ is $\mu$-Lipschitz via $\tilde{R}_{1}^{*}+\tilde{R}_{2}^{*}$.

The next theorem from Isaia is used to establish our desired results on the proposed mixed multi-order Caputo-Hadamard boundary problem (3).

Theorem 1 ([31]). Let $\Psi^{*}: \mathcal{W} \rightarrow \mathcal{W}$ be $\mu$-condensing and

$$
\mathfrak{G}^{*}=\left\{u \in \mathcal{W}: \exists \sigma \in[0,1], \text { so that } u=\sigma\left(\Psi^{*} u\right)\right\} .
$$

By assuming $\mathfrak{G}^{*}$ as a bounded subset belonging to $\mathcal{W}$, that is, $\tilde{m}>0$ exists so that $\mathfrak{G}^{*} \subset \overline{\mathbb{V}_{\tilde{m}}(0)}$, with $\overline{\mathbb{V}_{\tilde{m}}(0)}=\left\{u \in \mathcal{W}:\|u\|_{\mathcal{W}} \leq \tilde{m}\right\}$, then:

$$
\operatorname{Deg}\left(I-\sigma \Psi^{*}, \overline{\mathbb{V}_{\tilde{m}}(0)}, 0\right)=1 .
$$

In addition, $\Psi^{*}$ admits at least one fixed point and $\overline{\mathbb{V}_{\tilde{m}}(0)}$ involves all fixed points of $\Psi^{*}$.

The following theorem, due to Leray-Schauder, yields another criterion to establish the existence property for the suggested mixed multi-order Caputo-Hadamard-FBVP (3).

Theorem 2 ([32]). Let $\mathcal{N}$ be a convex, closed set in $\mathcal{W}, \mathcal{O}$ be open set belonging to $\mathcal{N}$, and $0 \in \mathcal{O}$. In addition, a function $\Psi^{*}: \overline{\mathcal{O}} \rightarrow \mathcal{N}$ is supposed to be continuous and compact.

In this case, either:

(L1) $\Psi^{*}$ admits a fixed point in $\overline{\mathcal{O}}$; or

(L2) $\exists u \in \partial \mathcal{O}, \exists \omega^{*} \in(0,1)$ s.t. $u=\omega^{*} \Psi^{*}(u)$. 


\section{Main Results}

After determining some of the preliminaries, we intended to reach our main goal for deriving analytical criteria that guarantee that the proposed mixed multi-order CaputoHadamard-FBVP (3) admits solution on $[1, M]$. First, we constructed the space: $\mathcal{W}=$ $\left\{u(t): u(t) \in C_{\mathbb{R}}([1, M])\right\}$, along with $\|u\|_{\mathcal{W}}=\sup _{t \in[1, M]}|u(t)|$. In this case, an ordered pair $\left(\mathcal{W},\|\cdot\|_{\mathcal{W}}\right)$ has all of the properties of a Banach space. Now, the first structural result is indicated in the following lemma to illustrate the framework of the equivalent integral solution for the mixed multi-order Caputo-Hadamard-FBVP (3).

Lemma 2. Let $\mathcal{Q} \in \mathcal{W}, \lambda, \mu_{1}^{*}, \mu_{2}^{*} \in(0,1], \gamma_{1}^{*}, \gamma_{2}^{*} \in\left(0, \varsigma-\theta^{*}\right)$ with $2<\theta^{*}<\varsigma<3$, $q_{1}^{*}, q_{2}^{*} \in \mathbb{R}^{+}, \delta_{1}, \delta_{2} \in \mathbb{R}$ and $\Theta^{*} \neq 0$. Then, $\hat{u}_{0}$ is a solution for the mixed multi-order Caputo-Hadamard-FBVP

$$
\left\{\begin{array}{l}
\lambda^{\mathcal{C H}} \mathfrak{D}_{1^{+}}^{\varsigma} u(t)+{ }^{\mathcal{C H}} \mathfrak{D}_{1^{+}}^{\theta^{*}} u(t)=\mathcal{Q}(t), \\
u(1)=0, \\
\mu_{1}^{* \mathcal{C H}} \mathfrak{D}_{1^{+}}^{\gamma_{1}^{*}} u(M)+{ }^{\mathcal{C H}} \mathfrak{D}_{1^{+}}^{\gamma_{2}^{*}} u(\eta)=\delta_{1}, \\
\mu_{2}^{* \mathcal{H}} \mathcal{I}_{1^{+}}^{q_{1}^{*}} u(M)+{ }^{\mathcal{H}} \mathcal{I}_{1^{+}}^{q_{2}^{*}} u(\eta)=\delta_{2},
\end{array}\right.
$$

iff $\hat{u}_{0}$ satisfies:

$$
\begin{aligned}
& \hat{u}_{0}(t)=\frac{1}{\Gamma(\varsigma)} \int_{1}^{t}\left(\ln \frac{t}{r}\right)^{\varsigma-1} \mathcal{Q}(r) \frac{\mathrm{d} r}{r}-\frac{1}{\Gamma\left(\varsigma-\theta^{*}\right)} \int_{1}^{t}\left(\ln \frac{t}{r}\right)^{\varsigma-\theta^{*}-1} \hat{u}_{0}(r) \frac{\mathrm{d} r}{r}
\end{aligned}
$$

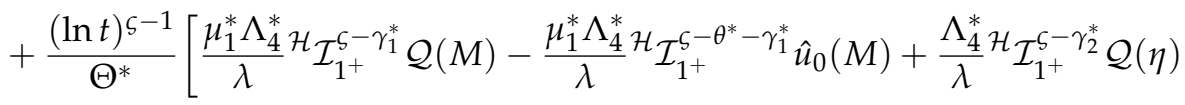

$$
\begin{aligned}
& -\frac{\Lambda_{4}^{*}}{\lambda} \mathcal{H}_{1^{+}}^{\zeta-\theta^{*}-\gamma_{2}^{*}} \hat{u}_{0}(\eta)-\frac{\mu_{2}^{*} \Lambda_{2}^{*}}{\lambda} \mathcal{H} \mathcal{I}_{1^{+}}^{\zeta+q_{1}^{*}} \mathcal{Q}(M)+\frac{\mu_{2}^{*} \Lambda_{2}^{*}}{\lambda} \mathcal{H} \mathcal{I}_{1^{+}}^{\varsigma+q_{1}^{*}-\theta^{*}} \hat{u}_{0}(M)-\frac{\Lambda_{2}^{*}}{\lambda} \mathcal{H} \mathcal{I}_{1^{+}}^{\zeta+q_{2}^{*}} \mathcal{Q}(\eta) \\
& \left.+\frac{\Lambda_{2}^{*}}{\lambda} \mathcal{H}_{1^{+}}^{\varsigma+q_{2}^{*}-\theta^{*}} \hat{u}_{0}(\eta)-\delta_{1} \Lambda_{4}^{*}+\delta_{2} \Lambda_{2}^{*}\right]+\frac{(\ln t)^{\zeta-2}}{\Theta^{*}}\left[\frac{-\mu_{1}^{*} \Lambda_{3}^{*}}{\lambda} \mathcal{H}_{\mathcal{I}^{\zeta+}}^{\zeta-\gamma_{1}^{*}} \mathcal{Q}(M)\right.
\end{aligned}
$$

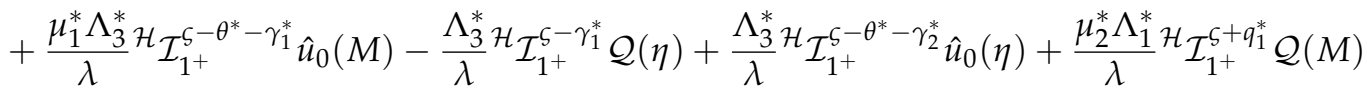

$$
\begin{aligned}
& \left.-\frac{\mu_{2}^{*} \Lambda_{1}^{*}}{\lambda} \mathcal{H}_{1^{+}}^{\varsigma+q_{1}^{*}-\theta^{*}} \hat{u}_{0}(M)+\frac{\Lambda_{1}^{*}}{\lambda} \mathcal{H}_{\mathcal{I}^{+}}^{\varsigma+q_{2}^{*}} \mathcal{Q}(\eta)-\frac{\Lambda_{1}^{*}}{\lambda} \mathcal{H}_{\mathcal{I}^{+}}^{\varsigma+q_{2}^{*}-\theta^{*}} \hat{u}_{0}(\eta)+\delta_{1} \Lambda_{3}^{*}-\delta_{2} \Lambda_{1}^{*}\right],
\end{aligned}
$$

so that:

$$
\begin{aligned}
& \Lambda_{1}^{*}=\mu_{1}^{*} \frac{\Gamma(\varsigma)}{\Gamma\left(\varsigma-\gamma_{1}^{*}\right)}(\ln M)^{\varsigma-\gamma_{1}^{*}-1}+\frac{\Gamma(\varsigma)}{\Gamma\left(\varsigma-\gamma_{2}^{*}\right)}(\ln \eta)^{\varsigma-\gamma_{2}^{*}-1}, \\
& \Lambda_{2}^{*}=\mu_{1}^{*} \frac{\Gamma(\varsigma)}{\Gamma\left(\varsigma-\gamma_{1}^{*}\right)}(\ln M)^{\varsigma-\gamma_{1}^{*}-2}+\frac{\Gamma(\varsigma)}{\Gamma\left(\varsigma-\gamma_{2}^{*}\right)}(\ln \eta)^{\varsigma-\gamma_{2}^{*}-2}, \\
& \Lambda_{3}^{*}=\mu_{2}^{*} \frac{\Gamma(\varsigma)}{\Gamma\left(\varsigma+q_{1}^{*}\right)}(\ln M)^{\left(\varsigma+q_{1}^{*}-1\right)}+\frac{\Gamma(\varsigma)}{\Gamma\left(\varsigma+q_{2}^{*}\right)}(\ln \eta)^{\left(\varsigma+q_{2}^{*}-1\right)}, \\
& \Lambda_{4}^{*}=\mu_{2}^{*} \frac{\Gamma(\varsigma)}{\Gamma\left(\varsigma+q_{1}^{*}\right)}(\ln M)^{\varsigma+q_{1}^{*}-2}+\frac{\Gamma(\varsigma)}{\Gamma\left(\varsigma+q_{2}^{*}\right)}(\ln \eta)^{\varsigma+q_{2}^{*}-2},
\end{aligned}
$$




$$
\Theta^{*}=\Lambda_{2}^{*} \Lambda_{3}^{*}-\Lambda_{1}^{*} \Lambda_{4}^{*}
$$

Proof. Suppose that $\hat{u}_{0}$ satisfies the given Caputo-Hadamard multi-order differential Equation (4). Then, we reach the following Hadamard integral equation:

$$
\hat{u}_{0}(t)=\frac{1}{\lambda} \mathcal{H}_{\mathcal{I}_{1^{+}}^{\varsigma}} \mathcal{Q}(t)-\frac{1}{\lambda} \mathcal{H}_{\mathcal{I}_{1^{+}}^{\varsigma-\theta^{*}}} \hat{u}_{0}(t)+p_{1}(\ln t)^{\varsigma-1}+p_{2}(\ln t)^{\varsigma-2}+p_{3}(\ln t)^{\varsigma-3} .
$$

We have to derive three unknowns, namely, $p_{1}, p_{2}$, and $p_{3} \in \mathbb{R}$ that appear in above integral equation. Since $2<\varsigma<3$, from the first boundary condition given in (4), we can obtain $p_{3}=0$. On the contrary, by utilizing the $\alpha^{* \text { th }}$-Caputo-Hadamard derivative and $q^{* t h}$-Hadamard integral so that $\alpha^{*} \in\left\{\gamma_{1}^{*}, \gamma_{2}^{*}\right\}, q * \in\left\{q_{1}^{*}, q_{2}^{*}\right\}$ and $0<\theta^{*}<\varsigma-\beta^{*}$, we have:

$$
\begin{aligned}
\mathcal{C H}_{\mathfrak{D}_{1+}^{\alpha^{*}} \hat{u}_{0}(t)} & =\frac{1}{\lambda} \mathcal{H}_{\mathcal{I}_{1^{+}}^{\varsigma-\alpha^{*}}} \mathcal{Q}(t)-\frac{1}{\lambda} \mathcal{H}_{\mathcal{I}^{+}}^{\varsigma-\theta^{*}-\alpha^{*}} \hat{u}_{0}(t) \\
& +p_{1} \frac{\Gamma(\varsigma)}{\Gamma\left(\varsigma-\alpha^{*}\right)}(\ln t)^{\left(\varsigma-\alpha^{*}-1\right)}+p_{2} \frac{\Gamma(\varsigma)}{\Gamma\left(\varsigma-\alpha^{*}\right)}(\ln t)^{\left(\varsigma-\alpha^{*}-2\right)}
\end{aligned}
$$

and:

$$
\begin{aligned}
\mathcal{H}_{\mathcal{I}^{+}}^{\beta^{*}} \hat{u}_{0}(t) & =\frac{1}{\lambda} \mathcal{H}_{\mathcal{I}_{1^{+}}^{\varsigma+\beta^{*}}} \mathcal{Q}(t)-\frac{1}{\lambda} \mathcal{H}_{\mathcal{I}_{1^{+}}^{\varsigma-\theta^{*}+\beta^{*}}} \hat{u}_{0}(t) \\
& +p_{1} \frac{\Gamma(k)}{\Gamma\left(\varsigma+\beta^{*}\right)}(\ln t)^{\left(\varsigma+\beta^{*}-1\right)}+p_{2} \frac{\Gamma(\varsigma)}{\Gamma\left(\varsigma+\beta^{*}\right)}(\ln t)^{\left(\varsigma+\beta^{*}-2\right)} .
\end{aligned}
$$

Inserting $\alpha^{*}=\gamma_{1}^{*}, \alpha^{*}=\gamma_{2}^{*}, \beta^{*}=q_{1}^{*}$, and $\beta^{*}=q_{2}^{*}$ into the above relations and invoking the second condition of (4), we reach:

$$
\begin{aligned}
p_{1} & =\frac{1}{\Lambda_{2}^{*} \Lambda_{3}^{*}-\Lambda_{1}^{*} \Lambda_{4}^{*}}\left[\frac{\mu_{1}^{*} \Lambda_{4}^{*}}{\lambda} \mathcal{H} I_{1^{+}}^{\zeta-\gamma_{1}^{*}} \mathcal{Q}(M)-\frac{\mu_{1}^{*} \Lambda_{4}^{*}}{\lambda} \mathcal{H} I_{1^{+}}^{\zeta-\theta^{*}-\gamma_{1}^{*}} \hat{u}_{0}(M)\right. \\
& +\frac{\Lambda_{4}^{*}}{\lambda} \mathcal{H} I_{1^{+}}^{\zeta-\gamma_{2}^{*}} \mathcal{Q}(\eta)-\frac{\Lambda_{4}^{*}}{\lambda} \mathcal{H} I_{1^{+}}^{\zeta-\theta^{*}-\gamma_{2}^{*}} \hat{u}_{0}(\eta)-\frac{\mu_{2}^{*} \Lambda_{2}^{*}}{\lambda} \mathcal{H} I_{1^{+}}^{\zeta+q_{1}^{*}} \mathcal{Q}(M)+\frac{\mu_{2}^{*} \Lambda_{2}^{*}}{\lambda} \mathcal{H} I_{1^{+}}^{\zeta+q_{1}^{*}-\theta^{*}} \hat{u}_{0}(M) \\
& \left.-\frac{\Lambda_{2}^{*}}{\lambda} \mathcal{H} I_{1^{+}}^{\zeta+q_{2}^{*}} \mathcal{Q}(\eta)+\frac{\Lambda_{2}^{*}}{\lambda} \mathcal{H} I_{1^{+}}^{\zeta+q_{2}^{*}-\theta^{*}} \hat{u}_{0}(\eta)-\delta_{1} \Lambda_{4}^{*}+\delta_{2} \Lambda_{2}^{*}\right]
\end{aligned}
$$

and:

$$
\begin{aligned}
p_{2} & =\frac{1}{\Lambda_{2}^{*} \Lambda_{3}^{*}-\Lambda_{1}^{*} \Lambda_{4}^{*}}\left[-\frac{\mu_{1}^{*} \Lambda_{3}^{*}}{\lambda} \mathcal{H} I_{1^{+}}^{\zeta-\gamma_{1}^{*}} \mathcal{Q}(M)+\frac{\mu_{1}^{*} \Lambda_{3}^{*}}{\lambda} \mathcal{H} I_{1^{+}}^{\zeta-\theta^{*}-\gamma_{1}^{*}} \hat{u}_{0}(M)\right. \\
& -\frac{\Lambda_{3}^{*}}{\lambda} \mathcal{H} I_{1^{+}}^{\zeta-\gamma_{2}^{*}} \mathcal{Q}(\eta)+\frac{\Lambda_{3}^{*}}{\lambda} \mathcal{H} I_{1^{+}}^{\zeta-\theta^{*}-\gamma_{2}^{*}} \hat{u}_{0}(\eta)+\frac{\mu_{2}^{*} \Lambda_{1}^{*}}{\lambda} \mathcal{H} I_{1^{+}}^{\zeta+q_{1}^{*}} \mathcal{Q}(M)+\frac{\mu_{2}^{*} \Lambda_{2}^{*} \mathcal{H} I_{1^{+}}^{\zeta+q_{1}^{*}-\theta^{*}} \hat{u}_{0}(M)}{} \\
& \left.+\frac{\Lambda_{1}^{*}}{\lambda} \mathcal{H} I_{1^{+}}^{\zeta+q_{2}^{*}} \mathcal{Q}(\eta)-\frac{\Lambda_{1}^{*}}{\lambda} \mathcal{H} I_{1^{+}}^{\zeta+q_{2}^{*}-\theta^{*}} \hat{u}_{0}(\eta)+\delta_{1} \Lambda_{3}^{*}-\delta_{2} \Lambda_{1}^{*}\right] .
\end{aligned}
$$

Eventually, we can insert the above-obtained values for unknown constants $p_{1}$ and $p_{2}$ into (6), and this means that $\hat{u}_{0}$ satisfies the Hadamard integral Equation (5). The converse is immediately followed by some simple calculations. This ends the proof.

To reach the main purpose for deriving the existence criteria for the solutions of the mixed multi-order Caputo-Hadamard boundary problem (3), in the light of Lemma 2, we formulated an operator $\Psi^{*}: \mathcal{W} \rightarrow \mathcal{W}$ as follows: 


$$
\begin{aligned}
& \Psi^{*} u(t)=\frac{1}{\Gamma(\varsigma)} \int_{1}^{t}\left(\ln \frac{t}{r}\right)^{\varsigma-1} \hat{A}(r, u(r)) \frac{\mathrm{d} r}{r}-\frac{1}{\Gamma\left(\varsigma-\theta^{*}\right)} \int_{1}^{t}\left(\ln \frac{t}{r}\right)^{\varsigma-\theta^{*}-1} u(r) \frac{\mathrm{d} r}{r} \\
& +\frac{(\ln t)^{\zeta-1}}{\Theta^{*}}\left[\frac{\mu_{1}^{*} \Lambda_{4}^{*}}{\lambda} \mathcal{H}_{\mathcal{I}^{\zeta}}^{\zeta-\gamma_{1}^{*}} \hat{A}(M, u(M))-\frac{\mu_{1}^{*} \Lambda_{4}^{*}}{\lambda} \mathcal{H} \mathcal{I}_{1^{+}}^{\zeta-\theta^{*}-\gamma_{1}^{*}} u(M)+\frac{\Lambda_{4}^{*}}{\lambda} \mathcal{H}_{\mathcal{I}^{\zeta}}^{\zeta-\gamma_{2}^{*}} \hat{A}(\eta, u(\eta))\right.
\end{aligned}
$$

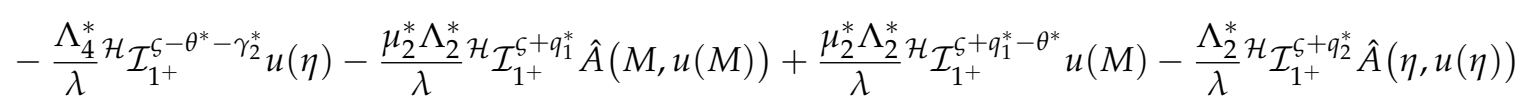

$$
\begin{aligned}
& \left.+\frac{\Lambda_{2}^{*}}{\lambda} \mathcal{H}_{1^{+}}^{\varsigma+q_{2}^{*}-\theta^{*}} u(\eta)-\delta_{1} \Lambda_{4}^{*}+\delta_{2} \Lambda_{2}^{*}\right]+\frac{(\ln t)^{\zeta-2}}{\Theta^{*}}\left[\frac{-\mu_{1}^{*} \Lambda_{3}^{*}}{\lambda} \mathcal{H}_{1^{+}}^{\zeta-\gamma_{1}^{*}} \hat{A}(M, u(M))\right. \\
& +\frac{\mu_{1}^{*} \Lambda_{3}^{*}}{\lambda} \mathcal{H} \mathcal{I}_{1^{+}}^{\zeta-\theta^{*}-\gamma_{1}^{*}} u(M)-\frac{\Lambda_{3}^{*}}{\lambda} \mathcal{H} \mathcal{I}_{1^{+}}^{\zeta-\gamma_{1}^{*}} \hat{A}(\eta, u(\eta))+\frac{\Lambda_{3}^{*}}{\lambda} \mathcal{H}_{1^{+}}^{\zeta-\theta^{*}-\gamma_{2}^{*}} u(\eta)+\frac{\mu_{2}^{*} \Lambda_{1}^{*}}{\lambda} \mathcal{H}_{\mathcal{I}^{+}}^{\zeta+q_{1}^{*}} \hat{A}(M, u(M)) \\
& \left.-\frac{\mu_{2}^{*} \Lambda_{1}^{*}}{\lambda} \mathcal{H}_{\mathcal{I}^{+}}^{\varsigma+q_{1}^{*}-\theta^{*}} u(M)+\frac{\Lambda_{1}^{*}}{\lambda} \mathcal{H}_{\mathcal{I}_{1^{+}}^{\zeta+q_{2}^{*}}} \hat{A}(\eta, u(\eta))-\frac{\Lambda_{1}^{*}}{\lambda} \mathcal{H}_{\mathcal{I}^{+}}^{\varsigma+q_{2}^{*}-\theta^{*}} u(\eta)+\delta_{1} \Lambda_{3}^{*}-\delta_{2} \Lambda_{1}^{*}\right]
\end{aligned}
$$

for each $u \in \mathcal{W}$. Before attempting to prove the first result, we first regarded some initial hypotheses that are utilized in the rest of the article.

$(\mathcal{S} 1)$ There is a constant, $\mathfrak{L}_{\hat{A}}>0$, so that for any $u_{1}, u_{2} \in \mathcal{W}$, the following inequality holds:

$$
\left|\hat{A}\left(t, u_{1}\right)-\hat{A}\left(t, u_{2}\right)\right| \leq \mathfrak{L}_{\hat{A}}\left|u_{1}-u_{2}\right| .
$$

$(\mathcal{S} 2)$ There exist two constants, $\mathfrak{C}_{\hat{A}}$ and $\mathfrak{M}_{\hat{A}}$, so that for any $u \in \mathcal{W}$ :

$$
|\hat{A}(t, u)| \leq \mathfrak{C}_{\hat{A}}|u|+\mathfrak{M}_{\hat{A}}
$$

(S3) Let $\mathfrak{L}_{\hat{A}} \mathcal{Q}^{*}<1$, so that:

$$
\begin{aligned}
\mathcal{Q}^{*} & =\frac{(\ln M)^{\varsigma}}{\lambda \Gamma(\varsigma+1)}+\left(\frac{(\ln M)^{\varsigma-1}\left|\Lambda_{4}^{*}\right|}{\lambda \Theta^{*}}+\frac{(\ln M)^{\varsigma-2}\left|\Lambda_{3}^{*}\right|}{\lambda \Theta^{*}}\right)\left[\frac{\left|\mu_{1}^{*}\right|(\ln M)^{\varsigma-\gamma_{1}^{*}}}{\Gamma\left(\varsigma-\gamma_{1}^{*}+1\right)}+\frac{(\ln M)^{\varsigma-\gamma_{2}^{*}}}{\Gamma\left(\varsigma-\gamma_{2}^{*}+1\right)}\right] \\
& +\left(\frac{(\ln M)^{\varsigma-1}\left|\Lambda_{2}^{*}\right|}{\lambda \Theta^{*}}+\frac{(\ln M)^{\varsigma-2}\left|\Lambda_{1}^{*}\right|}{\lambda \Theta^{*}}\right)\left[\frac{\left|\mu_{2}^{*}\right|(\ln M)^{\varsigma+q_{1}^{*}}}{\Gamma\left(\varsigma+q_{1}^{*}+1\right)}+\frac{(\ln M)^{\varsigma+q_{2}^{*}}}{\Gamma\left(\varsigma+q_{2}^{*}+1\right)}\right] .
\end{aligned}
$$

In addition, we split the operator $\Psi^{*}: \mathcal{W} \rightarrow \mathcal{W}$ into two operators, i.e., $\Psi_{1}^{*}: \mathcal{W} \rightarrow \mathcal{W}$ and $\Psi_{2}^{*}: \mathcal{W} \rightarrow \mathcal{W}$, by the following formulations:

$$
\begin{aligned}
& \left(\Psi_{1}^{*} u\right)(t)=\frac{1}{\Gamma(\varsigma)} \int_{1}^{t}\left(\ln \frac{t}{r}\right)^{\zeta-1} \hat{A}(r, u(r)) \frac{\mathrm{d} r}{r}
\end{aligned}
$$

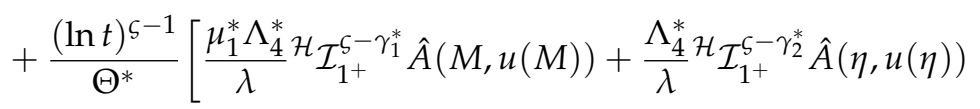

$$
\begin{aligned}
& \left.-\frac{\mu_{2}^{*} \Lambda_{2}^{*}}{\lambda} \mathcal{H} \mathcal{I}_{1^{+}}^{\zeta+q_{1}^{*}} \hat{A}(M, u(M))-\frac{\Lambda_{2}^{*}}{\lambda} \mathcal{H} \mathcal{I}_{1^{+}}^{\zeta+q_{2}^{*}} \hat{A}(\eta, u(\eta))\right] \\
& +\frac{(\ln t)^{\varsigma-2}}{\Theta^{*}}\left[-\frac{\mu_{1}^{*} \Lambda_{3}^{*}}{\lambda} \mathcal{H} \mathcal{I}_{1^{+}}^{\zeta-\gamma_{1}^{*}} \hat{A}(M, u(M))-\frac{\Lambda_{3}^{*}}{\lambda} \mathcal{H} \mathcal{I}_{1^{+}}^{\zeta-\gamma_{2}^{*}} \hat{A}(\eta, u(\eta))\right.
\end{aligned}
$$




$$
\left.+\frac{\mu_{2}^{*} \Lambda_{1}^{*}}{\lambda} \mathcal{I}_{1^{+}}^{\varsigma+q_{1}^{*}} \hat{A}(M, u(M))+\frac{\Lambda_{1}^{*}}{\lambda} \mathcal{H}_{\mathcal{I}^{+}}^{\varsigma+q_{2}^{*}} \hat{A}(\eta, u(\eta))\right]
$$

and:

$$
\begin{aligned}
& \left(\Psi_{2}^{*} u\right)(t)=\frac{1}{\Gamma\left(\varsigma-\theta^{*}\right)} \int_{1}^{t}\left(\ln \frac{t}{r}\right)^{\varsigma-\theta^{*}-1} u(r) \frac{\mathrm{d} r}{r}
\end{aligned}
$$

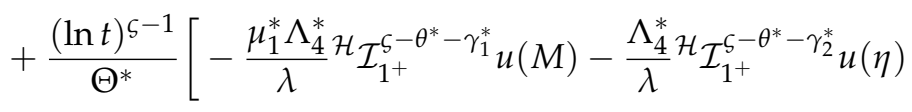

$$
\begin{aligned}
& \left.+\frac{\mu_{2}^{*} \Lambda_{2}^{*}}{\lambda} \mathcal{H} \mathcal{I}_{1^{+}}^{\varsigma+q_{1}^{*}-\theta^{*}} u(M)+\frac{\Lambda_{2}^{*}}{\lambda} \mathcal{H}_{\mathcal{I}_{1^{+}}^{\varsigma+q_{2}^{*}-\theta^{*}}} u(\eta)-\delta_{1} \Lambda_{4}^{*}+\delta_{2} \Lambda_{2}^{*}\right] \\
& +\frac{(\ln t)^{\varsigma-2}}{\Theta^{*}}\left[\frac{\mu_{1}^{*} \Lambda_{3}^{*}}{\lambda} \mathcal{H} \mathcal{I}_{1^{+}}^{\zeta-\theta^{*}-\gamma_{1}^{*}} u(M)+\frac{\Lambda_{3}^{*}}{\lambda} I_{1^{+}}^{\varsigma-\theta^{*}-\gamma_{2}^{*}} u(\eta)\right.
\end{aligned}
$$

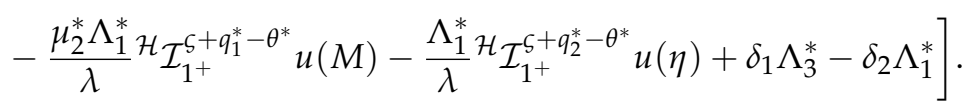

In fact, $\left(\Psi^{*} u\right)(t)=\left(\Psi_{1}^{*} u\right)(t)+\left(\Psi_{2}^{*} u\right)(t)$. In this position, it is evident that if there exists a fixed point for an operator $\Psi^{*}$ defined by $(7)$, then this is equivalent to the fact that there exists a solution for the mixed multi-order Caputo-Hadamard-FBVP (3).

Lemma 3. By taking into account hypothesis $(\mathcal{S} 1)$, the single-valued operator $\Psi_{1}^{*}: \mathcal{W} \rightarrow \mathcal{W}$ formulated above is $\mu$-Lipschitz with constant $\tilde{R}_{1}^{*}=\mathfrak{L}_{\hat{A}} \mathcal{Q}^{*}$, where $\mathcal{Q}^{*}$ is illustrated by (8), and we also have the growth condition $\left\|\Psi_{1}^{*}(u)\right\|_{\mathcal{W}} \leq \mathfrak{C}_{\hat{A}} \mathcal{Q}^{*}\|u\|_{\mathcal{W}}+\mathfrak{M}_{\hat{A}} \mathcal{Q}^{*}$ for any $u \in \mathcal{W}$.

Proof. Clearly, from hypothesis $(\mathcal{S} 1)$, we know that $\hat{A}$ is Lipschitz with constant $\mathfrak{L}_{\hat{A}}$. Thus, one may write:

$$
\begin{aligned}
& \left|\left(\Psi_{1}^{*} u\right)(t)-\left(\Psi_{1}^{*} u^{\prime}\right)(t)\right| \\
& \leq \frac{1}{\Gamma(\varsigma)} \int_{1}^{t}\left(\ln \frac{t}{r}\right)^{\varsigma-1}\left|\hat{A}(r, u(r))-\hat{A}\left(r, u^{\prime}(r)\right)\right| \frac{\mathrm{d} r}{r} \\
& +\frac{(\ln t)^{\varsigma-1}}{\left|\Theta^{*}\right|}\left[\frac{\left|\mu_{1}^{*}\right|\left|\Lambda_{4}^{*}\right|}{\lambda} \mathcal{H}_{1_{1^{+}}^{\varsigma-\gamma_{1}^{*}}}\left|\hat{A}(M, u(M))-\hat{A}\left(M, u^{\prime}(M)\right)\right|\right. \\
& +\frac{\left|\Lambda_{4}^{*}\right|}{\lambda} \mathcal{H}_{1_{1^{+}}^{\zeta-\gamma_{2}^{*}}}\left|\hat{A}(\eta, u(\eta))-\hat{A}\left(\eta, u^{\prime}(\eta)\right)\right| \\
& \left.+\frac{\left|\mu_{2}^{*}\right|\left|\Lambda_{2}^{*}\right|}{\lambda} \mathcal{H}_{\mathcal{I}_{1^{+}}^{\zeta+q_{1}^{*}}}\left|\hat{A}(M, u(M))-\hat{A}\left(M, u^{\prime}(M)\right)\right|+\frac{\left|\Lambda_{2}^{*}\right|}{\lambda} \mathcal{H}_{\mathcal{I}_{1^{+}}^{\zeta+q_{2}^{*}}}\left|\hat{A}(\eta, u(\eta))-\hat{A}\left(\eta, u^{\prime}(\eta)\right)\right|\right] \\
& +\frac{(\ln t)^{\varsigma-2}}{\left|\Theta^{*}\right|}\left[\frac{\left|\mu_{1}^{*}\right|\left|\Lambda_{3}^{*}\right|}{\lambda} \mathcal{H}_{1^{+}}^{\zeta-\gamma_{1}^{*}}\left|\hat{A}(M, u(M))-\hat{A}\left(M, u^{\prime}(M)\right)\right|\right. \\
& +\frac{\left|\Lambda_{3}^{*}\right|}{\lambda} \mathcal{H}_{1^{+}}^{\zeta-\gamma_{2}^{*}}\left|\hat{A}(\eta, u(\eta))-\hat{A}\left(\eta, u^{\prime}(\eta)\right)\right|+\frac{\left|\mu_{2}^{*}\right|\left|\Lambda_{1}^{*}\right|}{\lambda} \mathcal{H}_{\mathcal{I}_{1^{+}}^{\zeta+q_{1}^{*}}}\left|\hat{A}(M, u(M))-\hat{A}\left(M, u^{\prime}(M)\right)\right| \\
& \left.+\frac{\left|\Lambda_{1}^{*}\right|}{\lambda} \mathcal{H}_{1_{1^{+}}^{\zeta+q_{2}^{*}}}\left|\hat{A}(\eta, u(\eta))-\hat{A}\left(\eta, u^{\prime}(\eta)\right)\right|\right] \\
& \leq \mathfrak{L}_{\hat{A}}\left\|u-u^{\prime}\right\| \mathcal{W}\left[\frac{(\ln M)^{\varsigma}}{\lambda \Gamma(\varsigma+1)}+\left(\frac{(\ln M)^{\varsigma-1}\left|\Lambda_{4}^{*}\right|}{\lambda \Theta^{*}}+\frac{(\ln M)^{\varsigma-2}\left|\Lambda_{3}^{*}\right|}{\lambda \Theta^{*}}\right)\left[\frac{\left|\mu_{1}^{*}\right|(\ln M)^{\varsigma-\gamma_{1}^{*}}}{\Gamma\left(\varsigma-\gamma_{1}^{*}+1\right)}\right.\right.
\end{aligned}
$$




$$
\begin{aligned}
& \left.\left.+\frac{(\ln M)^{\varsigma-\gamma_{2}^{*}}}{\Gamma\left(\varsigma-\gamma_{2}^{*}+1\right)}\right]+\left(\frac{(\ln M)^{\varsigma-1}\left|\Lambda_{2}^{*}\right|}{\lambda \Theta^{*}}+\frac{(\ln M)^{\varsigma-2}\left|\Lambda_{1}^{*}\right|}{\lambda \Theta^{*}}\right)\left[\frac{\left|\mu_{2}^{*}\right|(\ln M)^{\varsigma+q_{1}^{*}}}{\Gamma\left(\varsigma+q_{1}^{*}+1\right)}+\frac{(\ln M)^{\varsigma+q_{2}^{*}}}{\Gamma\left(\varsigma+q_{2}^{*}+1\right)}\right]\right] \\
& =\mathfrak{L}_{\hat{A}} \mathcal{Q}^{*}\left\|u-u^{\prime}\right\|_{\mathcal{W}}=\tilde{R}_{1}^{*}\left\|u-u^{\prime}\right\|_{\mathcal{W}} .
\end{aligned}
$$

This illustrates that $\Psi_{1}^{*}$ is Lipschitz with constant $\tilde{R}_{1}^{*}=\mathfrak{L}_{\hat{A}} \mathcal{Q}^{*}$. Then, in view of Proposition 1 , we can find that $\Psi_{1}^{*}$ is $\mu$-Lipschitz via $\tilde{R}_{1}^{*}=\mathfrak{L}_{\hat{A}} \mathcal{Q}^{*}$, where $\mu$ stands for the Kuratowski's measure of non-compactness (KMNC). On the contrary, with due attention to hypothesis $(\mathcal{S} 1)$, we have:

$$
\left\|\Psi_{1}^{*}(u)\right\|_{\mathcal{W}} \leq \mathfrak{C}_{\hat{A}} \mathcal{Q}^{*}\|u\|_{\mathcal{W}}+\mathfrak{M}_{\hat{A}} \mathcal{Q}^{*}
$$

and finally, the proof process is finished.

Lemma 4. Assume that the condition $(\mathcal{S} 2)$ holds. Then, $\Psi_{2}^{*}: \mathcal{W} \rightarrow \mathcal{W}$ is continuous and the growth condition $\left\|\Psi_{2}^{*}(u)\right\|_{\mathcal{W}} \leq \mathcal{Z}^{*}\|u\|_{\mathcal{W}}+\Delta_{1}^{*}$ is valid for any $u \in \mathcal{W}$, so that:

$$
\Delta_{1}^{*}=\frac{(\ln M)^{\varsigma-1}}{\Theta^{*}}\left(\left|\delta_{1} \Lambda_{4}^{*}\right|+\left|\delta_{2} \Lambda_{2}^{*}\right|\right)+\frac{(\ln M)^{\varsigma-2}}{\Theta^{*}}\left(\left|\delta_{1} \Lambda_{3}^{*}\right|+\left|\delta_{2} \Lambda_{1}^{*}\right|\right)
$$

and:

$$
\begin{aligned}
\mathcal{Z}^{*} & =\frac{(\ln M)^{\varsigma-\theta^{*}}}{\lambda \Gamma\left(\varsigma-\theta^{*}+1\right)}+\left(\frac{(\ln M)^{\varsigma-1}\left|\Lambda_{4}^{*}\right|}{\lambda \Theta^{*}}+\frac{(\ln M)^{\varsigma-2}\left|\Lambda_{3}^{*}\right|}{\lambda \Theta^{*}}\right)\left[\frac{\left|\mu_{1}^{*}\right|(\ln M)^{\varsigma-\gamma_{1}^{*}-\theta^{*}}}{\Gamma\left(\varsigma-\gamma_{1}^{*}-\theta^{*}+1\right)}\right. \\
& \left.+\frac{(\ln M)^{\varsigma-\gamma_{2}^{*}-\theta^{*}}}{\Gamma\left(\varsigma-\gamma_{2}^{*}-\theta^{*}+1\right)}\right]+\left(\frac{(\ln M)^{\varsigma-1}\left|\Lambda_{2}^{*}\right|}{\lambda \Theta^{*}}+\frac{(\ln M)^{\varsigma-2}\left|\Lambda_{1}^{*}\right|}{\lambda \Theta^{*}}\right) \\
& \times\left[\frac{\left|\mu_{2}^{*}\right|(\ln M)^{\varsigma+q_{1}^{*}-\theta^{*}}}{\Gamma\left(\varsigma+q_{1}^{*}-\theta^{*}+1\right)}+\frac{(\ln M)^{\varsigma+q_{2}^{*}-\theta^{*}}}{\Gamma\left(\varsigma+q_{2}^{*}-\theta^{*}+1\right)}\right] .
\end{aligned}
$$

Proof. Let $\left\{u_{n}\right\}$ be a continuous sequence of functions defined on $[1, M]$, so that $\lim _{n \rightarrow \infty} u_{n}(t)=u(t)$. By taking into account the dominated convergence theorem attributed to Lebesgue, we arrive at:

$$
\begin{aligned}
& \lim _{n \rightarrow \infty} \Psi_{2}^{*} u_{n}(t)=\frac{1}{\Gamma\left(\varsigma-\theta^{*}\right)} \int_{1}^{t}\left(\ln \frac{t}{r}\right)^{\varsigma-\theta^{*}-1} \lim _{n \rightarrow \infty} u_{n}(r) \frac{\mathrm{d} r}{r} \\
& +\frac{(\ln t)^{\varsigma^{-1}}}{\Theta^{*}}\left[-\frac{\mu_{1}^{*} \Lambda_{4}^{*}}{\lambda} \mathcal{H}_{1^{+}}^{1^{-} \theta^{*}-\gamma_{1}^{*}} \lim _{n \rightarrow \infty} u_{n}(M)-\frac{\Lambda_{4}^{*}}{\lambda} \mathcal{H}_{1_{1^{+}}^{\zeta-\theta^{*}-\gamma_{2}^{*}}} \lim _{n \rightarrow \infty} u_{n}(\eta)\right. \\
& \left.+\frac{\mu_{2}^{*} \Lambda_{2}^{*}}{\lambda} \mathcal{H}_{\mathcal{I}_{1^{+}}^{\varsigma+q_{1}^{*}-\theta^{*}}} \lim _{n \rightarrow \infty} u_{n}(M)+\frac{\Lambda_{2}^{*}}{\lambda} \mathcal{H}_{\mathcal{I}_{1^{+}}^{\varsigma+q_{2}^{*}-\theta^{*}}} \lim _{n \rightarrow \infty} u_{n}(\eta)-\delta_{1} \Lambda_{4}^{*}+\delta_{2} \Lambda_{2}^{*}\right] \\
& +\frac{(\ln t)^{\varsigma-2}}{\Theta^{*}}\left[\frac{\mu_{1}^{*} \Lambda_{3}^{*}}{\lambda} \mathcal{H} \mathcal{I}_{1^{+}}^{\zeta-\theta^{*}-\gamma_{1}^{*}} \lim _{n \rightarrow \infty} u_{n}(M)+\frac{\Lambda_{3}^{*}}{\lambda} I_{1^{+}}^{\zeta-\theta^{*}-\gamma_{2}^{*}} \lim _{n \rightarrow \infty} u_{n}(\eta)\right. \\
& \left.-\frac{\mu_{2}^{*} \Lambda_{1}^{*}}{\lambda} \mathcal{H}_{\mathcal{I}_{1^{+}}^{\varsigma+q_{1}^{*}-\theta^{*}}} \lim _{n \rightarrow \infty} u_{n}(M)-\frac{\Lambda_{1}^{*}}{\lambda} \mathcal{H}_{\mathcal{I}_{1^{+}}^{\varsigma+q_{2}^{*}-\theta^{*}}} \lim _{n \rightarrow \infty} u_{n}(\eta)+\delta_{1} \Lambda_{3}^{*}-\delta_{2} \Lambda_{1}^{*}\right] \\
& =\Psi_{2}^{*} u(t)
\end{aligned}
$$


for all $t \in[1, M]$. Therefore we realize that $\Psi_{2}^{*} u_{n}(t) \rightarrow \Psi_{2}^{*} u(t)$ for all $t \in[1, M]$ whenever $n \rightarrow \infty$, and thus, $\Psi_{2}^{*}$ is continuous on $\overline{\mathbb{V}_{\tilde{m}}(0)}$. In the sequel, to check the growth condition for an operator $\Psi_{2}^{*}$, the assumption $(\mathcal{S} 2)$ considered, and we have:

$$
\begin{aligned}
& \left|\Psi_{2}^{*} u(t)\right| \leq \frac{1}{\Gamma\left(\varsigma-\theta^{*}\right)} \int_{1}^{t}\left(\ln \frac{t}{r}\right)^{\varsigma-\theta^{*}-1}|u(r)| \frac{\mathrm{d} r}{r} \\
& +\frac{(\ln M)^{\varsigma-1}}{\Theta^{*}}\left[\frac{\mu_{1}^{*} \Lambda_{4}^{*}}{\lambda} \mathcal{H}_{\mathcal{I}^{+}}^{\varsigma-\theta^{*}-\gamma_{1}^{*}}|u(M)|+\frac{\Lambda_{4}^{*}}{\lambda} \mathcal{H}_{\mathcal{I}_{1^{+}}^{\zeta-\theta^{*}}-\gamma_{2}^{*}}|u(\eta)|\right. \\
& \left.+\frac{\mu_{2}^{*} \Lambda_{2}^{*}}{\lambda} \mathcal{H}_{1^{+}}^{\varsigma+q_{1}^{*}-\theta^{*}}|u(M)|+\frac{\Lambda_{2}^{*}}{\lambda} \mathcal{H}_{\mathcal{I}_{1^{+}}^{\varsigma+q_{2}^{*}-\theta^{*}}}|u(\eta)|+\left|\delta_{1} \Lambda_{4}^{*}\right|+\left|\delta_{2} \Lambda_{2}^{*}\right|\right] \\
& +\frac{(\ln M)^{\varsigma-2}}{\Theta^{*}}\left[\frac{\mu_{1}^{*} \Lambda_{3}^{*}}{\lambda} \mathcal{H} \mathcal{I}_{1^{+}}^{\zeta-\theta^{*}-\gamma_{1}^{*}}|u(M)|+\frac{\Lambda_{3}^{*}}{\lambda} \mathcal{H}_{\mathcal{I}^{+}}^{\varsigma-\theta^{*}-\gamma_{2}^{*}}|u(\eta)|\right. \\
& \left.+\frac{\mu_{2}^{*} \Lambda_{1}^{*}}{\lambda} \mathcal{H}_{1^{+}}^{\varsigma+q_{1}^{*}-\theta^{*}}|u(M)|+\frac{\Lambda_{1}^{*}}{\lambda} \mathcal{H}_{\mathcal{I}_{1^{+}}^{\varsigma+q_{2}^{*}-\theta^{*}}}|u(\eta)|+\left|\delta_{1} \Lambda_{3}^{*}\right|+\left|\delta_{2} \Lambda_{1}^{*}\right|\right] \\
& \leq\|u\|_{\mathcal{W}} \mathcal{Z}^{*}+\frac{(\ln M)^{\varsigma-1}}{\Theta^{*}}\left(\left|\delta_{1} \Lambda_{4}^{*}\right|+\left|\delta_{2} \Lambda_{2}^{*}\right|\right)+\frac{(\ln M)^{\varsigma-2}}{\Theta^{*}}\left(\left|\delta_{1} \Lambda_{3}^{*}\right|+\left|\delta_{2} \Lambda_{1}^{*}\right|\right) \\
& =\mathcal{Z}^{*}\|u\|_{\mathcal{W}}+\Delta_{1}^{*},
\end{aligned}
$$

and we reach the desired conclusion.

Lemma 5. $\Psi_{2}^{*}: \mathcal{W} \rightarrow \mathcal{W}$ is compact, $\mu$-Lipschitz via $\tilde{R}_{2}^{*}=0$ by assuming $\mu$ as the KMNC.

Proof. We regard a bounded set $\mathfrak{G}^{*} \subset \overline{\mathbb{V}_{\tilde{m}}(0)}=\left\{u \in \mathcal{W}:\|u\|_{\mathcal{W}} \leq \tilde{m}\right\} \subseteq \mathcal{W}$ and an arbitrary sequence $\left\{u_{n}\right\}$ in $\mathfrak{G}^{*}$. In light of Lemma 4 , we have inequality: $\left\|\Psi_{2}^{*}\left(u_{n}\right)\right\|_{\mathcal{W}} \leq$ $\mathcal{Z}^{*} \tilde{m}+\Delta_{1}^{*}$ for every $u_{n} \in \mathfrak{G}^{*}$. This ensures the boundedness of $\Psi_{2}^{*}\left(\mathfrak{G}^{*}\right)$. Moreover, we claim that $\left\{\Psi_{2}^{*}\left(u_{n}\right)\right\}$ is equi-continuous for every $u_{n} \in \mathfrak{G}^{*} \subset \overline{\mathbb{V}_{\tilde{m}}(0)}$. To confirm this claim, we select $t_{1}, t_{2} \in[1, M]$ arbitrarily, such that $t_{1}<t_{2}$. Now, one can write:

$$
\begin{aligned}
& \left|\left(\Psi_{2}^{*} u_{n}\right)\left(t_{2}\right)-\left(\Psi_{2}^{*} u_{n}\right)\left(t_{1}\right)\right| \\
& \leq \tilde{m}\left|\frac{1}{\Gamma\left(\varsigma-\theta^{*}\right)} \int_{1}^{t_{2}}\left(\ln \frac{t_{2}}{r}\right)^{\varsigma-\theta^{*}-1} \frac{\mathrm{d} r}{r}-\frac{1}{\Gamma\left(\varsigma-\theta^{*}\right)} \int_{1}^{t_{1}}\left(\ln \frac{t_{1}}{r}\right)^{\varsigma-\theta^{*}-1} \frac{\mathrm{d} r}{r}\right|
\end{aligned}
$$

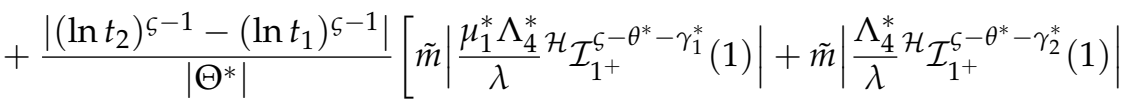

$$
\begin{aligned}
& \left.+\tilde{m}\left|\frac{\mu_{2}^{*} \Lambda_{2}^{*}}{\lambda} \mathcal{H}_{1^{+}}^{\zeta+q_{1}^{*}-\theta^{*}}(1)\right|+\tilde{m}\left|\frac{\Lambda_{2}^{*}}{\lambda} \mathcal{H}_{\mathcal{I}_{1^{+}}^{\zeta+q_{2}^{*}-\theta^{*}}}(1)\right|+\left|\delta_{1} \Lambda_{4}^{*}\right|+\left|\delta_{2} \Lambda_{2}^{*}\right|\right]
\end{aligned}
$$

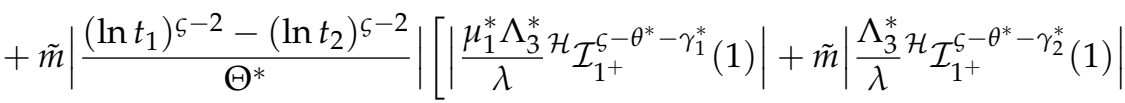

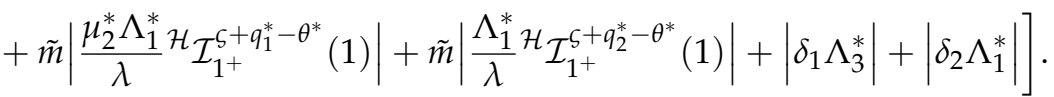

In view of above inequality, we can find that the RHS of the above relations approaches zero (free of $u_{n} \in \mathfrak{G}^{*}$ ) when we take $t_{1} \rightarrow t_{2}$. Thus, $\left|\Psi_{2}^{*}\left(u_{n}\right)\left(t_{2}\right)-\Psi_{2}^{*}\left(u_{n}\right)\left(t_{1}\right)\right|$ tends to 0 as $t_{1} \rightarrow t_{2}$. As a consequence, $\left\{\Psi_{2}^{*}\left(u_{n}\right)\right\}$ is equi-continuous, and with due attention to the Arzelà-Ascoli theorem, the compactness of $\Psi_{2}^{*}\left(\mathfrak{G}^{*}\right)$ is concluded. Eventually, by considering Proposition $2, \Psi_{2}^{*}$ is $\mu$-Lipschitz via $\tilde{R}_{2}^{*}=0$. 
Based on the above Lemmas, we intended to indicate the next theorem in which the existence criterion of the solution for the mixed multi-order Caputo-Hadamard boundary problem (3) is established.

Theorem 3. Suppose that the three hypotheses $(\mathcal{S} 1),(\mathcal{S} 2)$, and $(\mathcal{S} 3)$ are valid. Then, the proposed mixed multi-order Caputo-Hadamard boundary problem (3) has at least one solution $u \in \mathcal{W}$ if $\mathfrak{C}_{\hat{A}} \mathcal{Q}^{*}+\mathcal{Z}^{*}<1$. Moreover, the collection of all solutions of the multi-order problem (3) is a bounded set in $\mathcal{W}$.

Proof. With due attention to condition $(\mathcal{S} 3)$ and by Lemma 3, it is found that $\Psi_{1}^{*}: \mathcal{W} \rightarrow \mathcal{W}$ formulated in (9) has a $\mu$-Lipschitzian property with constant $\tilde{R}_{1}^{*}=\mathfrak{L}_{\hat{A}} \mathcal{Q}^{*} \in(0,1)$, where $\mathcal{Q}^{*}$ is illustrated by (8). On the contrary, by Lemma 5 , it is deduced that the operator $\Psi_{2}^{*}: \mathcal{W} \rightarrow \mathcal{W}$ formulated in (10) is $\mu$-Lipschitz with $\tilde{R}_{2}^{*}=0$. As a consequence, from Proposition 3, we confirm that the operator $\Psi^{*}: \mathcal{W} \rightarrow \mathcal{W}$ decomposed by $\Psi^{*}=\Psi_{1}^{*}+\Psi_{2}^{*}$ is a strict $\mu$-contraction via $\tilde{R}^{*}=\tilde{R}_{1}^{*}+\tilde{R}_{2}^{*}=\tilde{R}_{1}^{*}$. However, we know that $\tilde{R}^{*}=\tilde{R}_{1}^{*}<1$, so $\Psi^{*}$ is $\mu$-condensing. Subsequently, we constructed the following subset of $\mathcal{W}$ by:

$$
\mathfrak{G}^{*}:=\left\{u \in \mathcal{W}: \text { there exists } \sigma \in[0,1] \text {, so that } u=\sigma \Psi^{*}(u)\right\} .
$$

Here, we checked the boundedness of the subset $\mathfrak{G}^{*}$ in $\mathcal{W}$. To reach the desired purpose, we chose $u \in \mathfrak{G}^{*}$ arbitrarily. In this case, by considering the growth conditions achieved in both Lemmas 3 and 5, we obtained the following estimate:

$$
\begin{aligned}
\|u\|_{\mathcal{W}} & =\left\|\sigma \Psi^{*}(u)\right\|_{\mathcal{W}}=\sigma\left\|\Psi^{*}(u)\right\|_{\mathcal{W}} \leq \sigma\left(\left\|\Psi_{1}^{*}(u)\right\|_{\mathcal{W}}+\left\|\Psi_{2}^{*}(u)\right\|_{\mathcal{W}}\right) \\
& \leq \sigma\left(\mathfrak{C}_{\hat{A}} \mathcal{Q}^{*}\|u\|_{\mathcal{W}}+\mathfrak{M}_{\hat{A}} \mathcal{Q}^{*}+\mathcal{Z}^{*}\|u\|_{\mathcal{W}}+\Delta_{1}\right) \\
& \leq \sigma\left(\mathfrak{C}_{\hat{A}} \mathcal{Q}^{*}+\mathcal{Z}^{*}\right)\|u\|_{\mathcal{W}}+\sigma\left(\mathfrak{M}_{\hat{A}} \mathcal{Q}^{*}+\Delta_{1}^{*}\right) .
\end{aligned}
$$

The above estimates demonstrate that $\mathfrak{G}^{*}$ is bounded in $\mathcal{W}$. Hence, a positive number $\tilde{m}>0$ exists, so that $\mathfrak{G}^{*} \subset \overline{\mathbb{V}_{\tilde{m}}(0)}$, and thus, $\operatorname{Deg}\left(I-\sigma \Psi^{*}, \overline{\mathbb{V}_{\tilde{m}}(0)}, 0\right)=1$ according to Theorem 1. Eventually, since all items of Isaia's theorem 1 are valid, one can realize that at least one fixed point exists for the operator $\Psi^{*}=\Psi_{1}^{*}+\Psi_{2}^{*}$, and that the collection of all fixed points of $\Psi^{*}$ is a bounded subset in $\mathcal{W}$. From this, we understand that at least one solution to $[1, M]$ exists for the mixed multi-order Caputo-Hadamard boundary problem (3), and that the collection of all solutions is a bounded set-and so the proof is ended.

In the subsequent step, the uniqueness property of solution for the mixed multi-order Caputo-Hadamard-FBVP (3) is obtained in the next theorem.

Theorem 4. Suppose that all three assumptions $(\mathcal{S} 1),(\mathcal{S} 2)$, and $(\mathcal{S} 3)$ are valid, and that we also have the inequality $\mathfrak{L}_{\hat{A}} \mathcal{Q}^{*}+\mathcal{Z}^{*}<1$, where $\mathcal{Q}^{*}$ and $\mathcal{Z}^{*}$ are illustrated by (8) and (12), respectively. Then, a unique solution exists for $[1, M]$ for the mixed multi-order Caputo-Hadamard boundary problem (3).

Proof. To implement the deduction, we invoked the contraction principle due to Banach. By choosing $u \in \mathcal{W}$ arbitrarily and in view of Lemma 3 and condition $(\mathcal{S} 1)$, we have:

$$
\left|\Psi_{1}^{*} u(t)-\Psi_{1}^{*} u^{\prime}(t)\right| \leq \mathfrak{L}_{\hat{A}} \mathcal{Q}^{*}\left\|u-u^{\prime}\right\|_{\mathcal{W}}
$$

so that an operator $\Psi_{1}^{*}: \mathcal{W} \rightarrow \mathcal{W}$ is formulated by (9). In addition, we estimate:

$$
\begin{aligned}
& \left|\Psi_{2}^{*} u(t)-\Psi_{2}^{*} u^{\prime}(t)\right| \\
& \leq \frac{1}{\Gamma\left(\varsigma-\theta^{*}\right)} \int_{1}^{t}\left(\ln \frac{t}{r}\right)^{\varsigma-\theta^{*}-1}\left|u(r)-u^{\prime}(r)\right| \frac{\mathrm{d} r}{r}
\end{aligned}
$$




$$
\begin{aligned}
& +\frac{(\ln M)^{\varsigma-1}}{\Theta^{*}}\left[\frac{\mu_{1}^{*} \Lambda_{4}^{*}}{\lambda} \mathcal{H}_{1^{+}}^{\zeta-\theta^{*}-\gamma_{1}^{*}}\left|u(M)-u^{\prime}(M)\right|-\frac{\Lambda_{4}^{*}}{\lambda} \mathcal{H}_{1^{+}}{ }^{\zeta-\theta^{*}-\gamma_{2}^{*}}\left|u(\eta)-u^{\prime}(\eta)\right|\right. \\
& \left.+\frac{\mu_{2}^{*} \Lambda_{2}^{*}}{\lambda} \mathcal{H} \mathcal{I}_{1^{+}}^{\varsigma+q_{1}^{*}-\theta^{*}}\left|u(M)-u^{\prime}(M)\right|+\frac{\Lambda_{2}^{*}}{\lambda} \mathcal{H}_{\mathcal{I}_{1^{+}}^{\varsigma+q_{2}^{*}-\theta^{*}}}\left|u(\eta)-u^{\prime}(\eta)\right|\right] \\
& +\frac{(\ln M)^{\varsigma^{-2}}}{\Theta^{*}}\left[\frac{\mu_{1}^{*} \Lambda_{3}^{*}}{\lambda} \mathcal{H}_{\mathcal{I}_{1^{+}}^{\zeta-\theta^{*}-\gamma_{1}^{*}}}\left|u(M)-u^{\prime}(M)\right|+\frac{\Lambda_{3}^{*}}{\lambda} I_{1^{+}}^{\zeta-\theta^{*}-\gamma_{2}^{*}}\left|u(\eta)-u^{\prime}(\eta)\right|\right. \\
& \left.+\frac{\mu_{2}^{*} \Lambda_{1}^{*}}{\lambda} \mathcal{H}_{\mathcal{I}_{1^{+}}^{\varsigma+q_{1}^{*}-\theta^{*}}}\left|u(M)-u^{\prime}(M)\right|+\frac{\Lambda_{1}^{*}}{\lambda} \mathcal{H}_{1^{+}}^{\zeta+q_{2}^{*}-\theta^{*}}\left|u(\eta)-u^{\prime}(\eta)\right|\right] \\
& \leq \mathcal{Z}^{*}\left\|u-u^{\prime}\right\|_{\mathcal{W}}
\end{aligned}
$$

so that an operator $\Psi_{2}^{*}: \mathcal{W} \rightarrow \mathcal{W}$ is formulated by (10). As a consequence, by (13) and (14), we find that:

$$
\left\|\Psi^{*}(u)-\Psi^{*}\left(u^{\prime}\right)\right\|_{\mathcal{W}} \leq\left(\mathfrak{L}_{\hat{A}} \mathcal{Q}^{*}+\mathcal{Z}^{*}\right)\left\|u-u^{\prime}\right\|_{\mathcal{W}} .
$$

From the obtained result, we realize that $\Psi^{*}=\Psi_{1}^{*}+\Psi_{2}^{*}: \mathcal{W} \rightarrow \mathcal{W}$ is a contraction. Then, a unique solution exists for $[1, M]$ for the mixed multi-order Caputo-Hadamard boundary problem (3) according to the Banach principle, and this ends the proof.

In the final step, another existence criterion of the solution for the mixed multi-order Caputo-Hadamard boundary problem (3) is derived by invoking a result due to Leray-Schauder.

Theorem 5. Assume that $\mathcal{Z}^{*}<1$. Let $\hat{A}:[1, M] \times \mathcal{W} \rightarrow \mathcal{W}$ be continuous and a nondecreasing continuous mapping $\Phi_{1}:[0, \infty) \rightarrow(0, \infty)$ and $\Phi_{2} \in \mathcal{C}_{\mathbb{R}^{+}}([1, M])$, so that $|\hat{A}(t, u)| \leq$ $\Phi_{2}(t) \Phi_{1}\left(\|u\|_{\mathcal{W}}\right)$ for any $(t, u) \in[1, M] \times \mathcal{W}$. Furthermore, suppose that a constant $\mathfrak{N}^{*}>0$ exists, provided that:

$$
\frac{\left(1-\mathcal{Z}^{*}\right) \mathfrak{N}^{*}}{\Phi_{1}\left(\mathfrak{N}^{*}\right)\left\|\Phi_{2}\right\| \mathcal{Q}^{*}+\Delta_{1}^{*}}>1,
$$

where $\mathcal{Q}^{*}, \Delta_{1}^{*}$, and $\mathcal{Z}^{*}$ are illustrated by (8), (11), and (12), respectively. Then, at least one solution is found for $[1, M]$ for the mixed multi-order Caputo-Hadamard boundary problem (3).

Proof. To begin the proof, we first regard the operator $\Psi^{*}: \mathcal{W} \rightarrow \mathcal{W}$ formulated by (7). Now, we intend to check this property that every bounded set corresponds to bounded subsets of $\mathcal{W}$ by the operator $\Psi^{*}$. To confirm this subject, we take $\tilde{m}>0$ and $\overline{\mathbb{V}_{\tilde{m}}(0)}=$ $\left\{u \in \mathcal{W}:\|u\|_{\mathcal{W}} \leq \tilde{m}\right\}$ in the Banach space $\mathcal{W}$. In this case, for any $t \in[1, M]$ :

$$
\begin{aligned}
& \left|\Psi^{*} u(t)\right| \leq \sup _{t \in[1, M]} \mid \frac{1}{\Gamma(\varsigma)} \int_{1}^{t}\left(\ln \frac{t}{r}\right)^{\varsigma-1} \hat{A}(r, u(r)) \frac{\mathrm{d} r}{r}-\frac{1}{\Gamma\left(\varsigma-\theta^{*}\right)} \int_{1}^{t}\left(\ln \frac{t}{r}\right)^{\varsigma-\theta^{*}-1} u(r) \frac{\mathrm{d} r}{r} \\
& +\frac{(\ln t)^{\zeta-1}}{\Theta^{*}}\left[\frac{\mu_{1}^{*} \Lambda_{4}^{*}}{\lambda} \mathcal{H}_{\mathcal{I}_{1^{+}}^{\zeta-\gamma_{1}^{*}}} \hat{A}(M, u(M))-\frac{\mu_{1}^{*} \Lambda_{4}^{*}}{\lambda} \mathcal{H}_{\mathcal{I}^{+}}{ }^{\zeta-\theta^{*}-\gamma_{1}^{*}} u(M)+\frac{\Lambda_{4}^{*}}{\lambda} \mathcal{H}_{1^{+}}^{\zeta-\gamma_{2}^{*}} \hat{A}(\eta, u(\eta))\right. \\
& -\frac{\Lambda_{4}^{*}}{\lambda} \mathcal{H} \mathcal{I}_{1^{+}}^{\zeta-\theta^{*}-\gamma_{2}^{*}} u(\eta)-\frac{\mu_{2}^{*} \Lambda_{2}^{*}}{\lambda} \mathcal{H} \mathcal{I}_{1^{+}}^{\zeta+q_{1}^{*}} \hat{A}(M, u(M))+\frac{\mu_{2}^{*} \Lambda_{2}^{*}}{\lambda} \mathcal{H} \mathcal{I}_{1^{+}}^{\zeta+q_{1}^{*}-\theta^{*}} u(M)-\frac{\Lambda_{2}^{*}}{\lambda} \mathcal{H} \mathcal{I}_{1^{+}}^{\zeta+q_{2}^{*}} \hat{A}(\eta, u(\eta)) \\
& \left.+\frac{\Lambda_{2}^{*}}{\lambda} \mathcal{H}_{1^{+}}^{\zeta+q_{2}^{*}-\theta^{*}} u(\eta)-\delta_{1} \Lambda_{4}^{*}+\delta_{2} \Lambda_{2}^{*}\right]+\frac{(\ln t)^{\zeta-2}}{\Theta^{*}}\left[\frac{-\mu_{1}^{*} \Lambda_{3}^{*}}{\lambda} \mathcal{H}_{1^{+}}^{\zeta-\gamma_{1}^{*}} \hat{A}(M, u(M))\right. \\
& +\frac{\mu_{1}^{*} \Lambda_{3}^{*}}{\lambda} \mathcal{H} \mathcal{I}_{1^{+}}^{\zeta-\theta^{*}-\gamma_{1}^{*}} u(M)-\frac{\Lambda_{3}^{*}}{\lambda} \mathcal{H} \mathcal{I}_{1^{+}}^{\zeta-\gamma_{1}^{*}} \hat{A}(\eta, u(\eta))+\frac{\Lambda_{3}^{*}}{\lambda} \mathcal{H} \mathcal{I}_{1^{+}}^{\zeta-\theta^{*}-\gamma_{2}^{*}} u(\eta)+\frac{\mu_{2}^{*} \Lambda_{1}^{*}}{\lambda} \mathcal{H} \mathcal{I}_{1^{+}}^{\zeta+q_{1}^{*}} \hat{A}(M, u(M))
\end{aligned}
$$




$$
\begin{aligned}
& \left.-\frac{\mu_{2}^{*} \Lambda_{1}^{*}}{\lambda} \mathcal{H}_{\mathcal{I}^{+}}^{\varsigma+q_{1}^{*}-\theta^{*}} u(M)+\frac{\Lambda_{1}^{*}}{\lambda} \mathcal{H}_{\mathcal{I}_{1^{+}}^{\varsigma+q_{2}^{*}}} \hat{A}(\eta, u(\eta))-\frac{\Lambda_{1}^{*}}{\lambda} \mathcal{H}_{\mathcal{I}_{1^{+}}^{\varsigma+q_{2}^{*}-\theta^{*}}} u(\eta)+\delta_{1} \Lambda_{3}^{*}-\delta_{2} \Lambda_{1}^{*}\right] \mid \\
& \leq\left\|\Phi_{2}\right\|_{\mathcal{W}} \Phi_{1}\left(\|u\|_{\mathcal{W}}\right) \mathcal{Q}^{*}+\|u\|_{\mathcal{W}} \mathcal{Z}^{*}+\Delta_{1}^{*}
\end{aligned}
$$

and consequently:

$$
\left\|\Psi^{*}(u)\right\|_{\mathcal{W}} \leq\left\|\Phi_{2}\right\|_{\mathcal{W}} \Phi_{1}(\tilde{m}) \mathcal{Q}^{*}+\tilde{m} \mathcal{Z}^{*}+\Delta_{1}^{*}
$$

In the sequel, we continue the proof process to guarantee that every bounded set (balls) corresponds to equi-continuous subsets of $\mathcal{W}$ by the operator $\Psi^{*}$. By taking $m_{1}, m_{2} \in[1, M]$ with $m_{1}<m_{2}$ and $u \in \overline{\mathbb{V}_{\tilde{m}}(0)}$, we have:

$$
\begin{aligned}
& \left|\Psi^{*}\left(m_{2}\right)-\Psi^{*}\left(m_{1}\right)\right| \\
& \leq\left\|\Phi_{2}\right\|_{\mathcal{W}} \Phi_{1}(\tilde{m})\left|\frac{1}{\Gamma(\varsigma)} \int_{1}^{m_{2}}\left(\ln \frac{m_{2}}{r}\right)^{\varsigma-1} \frac{\mathrm{d} r}{r}-\frac{1}{\Gamma(\varsigma)} \int_{1}^{m_{1}}\left(\ln \frac{m_{1}}{r}\right)^{\varsigma^{-1}} \frac{\mathrm{d} r}{r}\right| \\
& +\tilde{m}\left|\frac{1}{\Gamma\left(\varsigma-\theta^{*}\right)} \int_{1}^{m_{2}}\left(\ln \frac{m_{2}}{r}\right)^{\varsigma-\theta^{*}-1} \frac{\mathrm{d} r}{r}-\frac{1}{\Gamma\left(\varsigma-\theta^{*}\right)} \int_{1}^{m_{1}}\left(\ln \frac{m_{1}}{r}\right)^{\varsigma-\theta^{*}-1} \frac{\mathrm{d} r}{r}\right| \\
& +\frac{\left|\left(\ln m_{2}\right)^{\varsigma-1}-\left(\ln m_{1}\right)^{\varsigma-1}\right|}{\left|\Theta^{*}\right|}\left\{\tilde{m}\left|\frac{\mu_{1}^{*} \Lambda_{4}^{*}}{\lambda} \mathcal{H}_{1^{+}}{ }^{\zeta-\theta^{*}-\gamma_{1}^{*}}(1)\right|+\tilde{m}\left|\frac{\Lambda_{4}^{*}}{\lambda} \mathcal{H}_{1^{+}}^{\varsigma-\theta^{*}-\gamma_{2}^{*}}(1)\right|\right. \\
& +\tilde{m}\left|\frac{\mu_{2}^{*} \Lambda_{2}^{*}}{\lambda} \mathcal{H}_{1^{+}}^{\zeta+q_{1}^{*}-\theta^{*}}(1)\right|+\tilde{m}\left|\frac{\Lambda_{2}^{*}}{\lambda} \mathcal{H}_{1^{+}}^{\zeta+q_{2}^{*}-\theta^{*}}(1)\right|\left\|\Phi_{2}\right\|_{\mathcal{W}} \Phi_{1}(\tilde{m})\left|\frac{\mu_{1}^{*} \Lambda_{4}^{*}}{\lambda} \mathcal{H}_{\mathcal{I}^{+}}^{\zeta-\gamma_{1}^{*}}(1)\right| \\
& +\left\|\Phi_{2}\right\|_{\mathcal{W}} \Phi_{1}(\tilde{m})\left|\frac{\Lambda_{4}^{*}}{\lambda} \mathcal{H}_{\mathcal{I}_{1^{+}}^{\zeta-\gamma_{2}^{*}}}(1)\right|+\left\|\Phi_{2}\right\|_{\mathcal{W}} \Phi_{1}(\tilde{m})\left|\frac{\mu_{2}^{*} \Lambda_{2}^{*}}{\lambda} \mathcal{H}_{\mathcal{I}^{+}}^{\varsigma+q_{1}^{*}}(1)\right|+\left\|\Phi_{2}\right\|_{\mathcal{W}} \Phi_{1}(\tilde{m})\left|\frac{\Lambda_{2}^{*}}{\lambda} \mathcal{H}_{\mathcal{I}_{1^{+}}^{\zeta+q_{2}^{*}}}(1)\right| \\
& \left.+\left|\delta_{1} \Lambda_{4}^{*}\right|+\left|\delta_{2} \Lambda_{2}^{*}\right|\right\}+\left|\frac{\left(\ln m_{1}\right)^{\varsigma-2}-\left(\ln m_{2}\right)^{\varsigma-2}}{\Theta^{*}}\right|\left\{\tilde{m}\left|\frac{\mu_{1}^{*} \Lambda_{3}^{*}}{\lambda} \mathcal{H}_{1^{+}}^{\zeta-\theta^{*}-\gamma_{1}^{*}}(1)\right|+\tilde{m}\left|\frac{\Lambda_{3}^{*}}{\lambda} \mathcal{H}_{1^{+}}^{\zeta-\theta^{*}-\gamma_{2}^{*}}(1)\right|\right. \\
& +\tilde{m}\left|\frac{\mu_{2}^{*} \Lambda_{1}^{*}}{\lambda} \mathcal{H}_{\mathcal{I}^{+}}^{\varsigma+q_{1}^{*}-\theta^{*}}(1)\right|+\tilde{m}\left|\frac{\Lambda_{1}^{*}}{\lambda} \mathcal{H}_{1^{+}}^{\varsigma+q_{2}^{*}-\theta^{*}}(1)\right|+\left\|\Phi_{2}\right\|_{\mathcal{W}} \Phi_{1}(\tilde{m})\left|\frac{\mu_{1}^{*} \Lambda_{3}^{*}}{\lambda} \mathcal{H}_{\mathcal{I}_{1+}^{\zeta-\gamma_{1}^{*}}}(1)\right| \\
& +\left\|\Phi_{2}\right\|{ }_{\mathcal{W}} \Phi_{1}(\tilde{m})\left|\frac{\Lambda_{3}^{*}}{\lambda} \mathcal{H}_{\mathcal{I}_{1^{+}}^{\zeta-\gamma_{2}^{*}}}(1)\right|+\left\|\Phi_{2}\right\|_{\mathcal{W}} \Phi_{1}(\tilde{m})\left|\frac{\mu_{2}^{*} \Lambda_{1}^{*}}{\lambda} \mathcal{H}_{\mathcal{I}_{1^{+}}^{\zeta+q_{1}^{*}}}(1)\right| \\
& \left.+\left\|\Phi_{2}\right\|{ }_{\mathcal{W}} \Phi_{1}(\tilde{m})\left|\frac{\Lambda_{1}^{*}}{\lambda} \mathcal{H}_{\mathcal{I}^{+}}^{\varsigma+q_{2}^{*}}(1)\right|+\left|\delta_{1} \Lambda_{3}^{*}\right|+\left|\delta_{2} \Lambda_{1}^{*}\right|\right\} .
\end{aligned}
$$

In view of above inequality, we can find that the RHS of above relations approaches zero (not depending on $\left.u \in \overline{\mathbb{V}_{\tilde{m}}(0)}\right)$ as $m_{1} \rightarrow m_{2}$. Thus, $\left|\Psi^{*}(u)\left(m_{2}\right)-\Psi^{*}(u)\left(m_{1}\right)\right|$ tends to 0 . Accordingly, $\left\{\Psi^{*}(u)\right\}$ is equi-continuous and with due attention to the Arzelà-Ascoli theorem, $\Psi^{*}\left(\overline{\mathbb{V}_{\tilde{m}}(0)}\right)$ is compact. The final considered goal in this proof is achieved by the help of the Leray-Schauder result once we conclude the boundedness of the collection of all solutions of $\omega^{*} \Psi^{*} u=u$ by choosing $\omega^{*} \in(0,1)$. To arrive at such an aim, we regard $u$ as having satisfied the mentioned equation. For each $t \in[1, M]$ and by some simple calculations, we obtain:

$$
|u(t)|=\left|\omega^{*}\right|\left|\Psi^{*} u(t)\right| \leq\left\|\Phi_{2}\right\| \Phi_{1}\left(\|u\|_{\mathcal{W}}\right) \mathcal{Q}^{*}+\|u\|_{\mathcal{W}} \mathcal{Z}^{*}+\Delta_{1}^{*}
$$


and thus it becomes:

$$
\frac{\left(1-\mathcal{Z}^{*}\right)\|u\|_{\mathcal{W}}}{\Phi_{1}\left(\|u\|_{\mathcal{W}}\right)\left\|\Phi_{2}\right\| \mathcal{Q}^{*}+\Delta_{1}^{*}}<1
$$

Based on the hypothesis, we select $\mathfrak{N}^{*} \in \mathbb{R}_{+}$, so that $\mathfrak{N}^{*} \neq\|u\|_{\mathcal{W}}$. Then we construct an open set $\mathcal{O}=\left\{u \in \mathcal{W}:\|u\|<\mathfrak{N}^{*}\right\}$. Then, we can easily realize that $\Psi^{*}: \overline{\mathcal{O}} \rightarrow \mathcal{W}$ is continuous and completely continuous. With due attention to such a choice of $\mathcal{O}$, there is no element $u \in \partial \mathcal{O}$ fulfilling $\omega^{*} \Psi^{*} u=u$ for one $\omega^{*} \in(0,1)$. Hence, based on the conditions of Theorem 2, it is followed that $\Psi^{*}$ possesses a fixed point $u \in \overline{\mathcal{O}}$, and accordingly, it is found a solution for the mixed multi-order Caputo-Hadamard-FBVP (3) on $[1, M]$, and the proof process is ended.

\section{Examples}

In this part of the current article, our analytical findings are supported by demonstrating two simulation examples in the numerical setting to indicate the applicability of our proofs.

Example 1. By taking into account the problem (3), we formulated the mixed multi-order CaputoHadamard fractional boundary problem:

$$
\left\{\begin{array}{l}
0.99^{\mathcal{C H}} \mathfrak{D}_{1^{+}}^{2.68} u(t)+{ }^{\mathcal{C H}} \mathfrak{D}_{1^{+}}^{2.01} u(t)=\frac{1}{49+\exp \left(t^{2}-1\right)}\left(\frac{|u(t)|}{25+|u(t)|}\right)+\frac{2020}{2021}, \\
u(1)=0, \quad 0.01^{\mathcal{C H}} \mathfrak{D}_{1^{+}}^{0.6} u\left(\frac{6}{5}\right)+{ }^{\mathcal{C H}} \mathfrak{D}_{1^{+}}^{0.5} u(1.01)=\frac{1}{5}, \\
0.99^{\mathcal{H}} \mathcal{I}_{1^{+}}^{0.05} u\left(\frac{6}{5}\right)+{ }^{\mathcal{H}} \mathcal{I}_{1^{+}}^{5.11} u(1.01)=\frac{1}{12} .
\end{array}\right.
$$

Here, $t \in\left[1, \frac{6}{5}\right], \lambda=0.99, \varsigma=2.68, \theta^{*}=2.01, \gamma_{1}^{*}=0.6, \gamma_{2}^{*}=0.5, q_{1}^{*}=0.5$, $q_{2}^{*}=5.11, \delta_{1}=\frac{1}{16}, \delta_{2}=\frac{5}{12}, \mu_{1}^{*}=0.01, \mu_{2}^{*}=0.99, \eta=1.01$, and $M=\frac{6}{5}$. Note that: $\gamma_{1}^{*}, \gamma_{2}^{*}<0.67=\varsigma-\theta^{*}$. Based on the above parameters, we can find that:

$$
\begin{aligned}
& \Lambda_{1}^{*} \simeq 0.0119, \quad \Lambda_{2}^{*} \simeq 0.9774, \quad \Lambda_{3}^{*} \simeq 0.0501, \quad \Lambda_{4}^{*} \simeq 0.2746, \\
& \Theta^{*} \simeq 0.0457, \quad \mathcal{Q}^{*} \simeq 0.0128, \quad \mathcal{Z}^{*} \simeq 0.9927 .
\end{aligned}
$$

In addition, we regarded $\hat{A}:\left[1, \frac{6}{5}\right] \times \mathbb{R} \rightarrow \mathbb{R}$ based on the following formulation:

$$
\hat{A}(t, u(t))=\frac{1}{49+\exp \left(t^{2}-1\right)}\left(\frac{|u(t)|}{25+|u(t)|}\right)+\frac{2020}{2021} .
$$

In this case, we obviously have:

$$
\left|\hat{A}(t, u(t))-\hat{A}\left(t, u^{\prime}(t)\right)\right| \leq \frac{1}{2}\left|u(t)-u^{\prime}(t)\right|
$$

and:

$$
|\hat{A}(t, u(t))| \leq \frac{1}{50}|u(t)|+\frac{2020}{2021}
$$

so that $\mathfrak{L}_{\hat{A}}=\frac{1}{2}, \mathfrak{C}_{\hat{A}}=\frac{1}{50}$ and $\mathfrak{M}_{\hat{A}}=\frac{2020}{2021}$. On the contrary, we obtained the constants:

$$
\tilde{R}_{1}^{*}=\mathfrak{L}_{\hat{A}} \mathcal{Q}^{*} \simeq 0.0064<1 \quad \text { and } \quad \mathfrak{C}_{\hat{A}} \mathcal{Q}^{*}+\mathcal{Z}^{*} \simeq 0.9930<1 .
$$


As a consequence, in view of Theorem 3, we realized that the mixed multi-order CaputoHadamard-FBVP (16) has at least a solution function u belonging to $\mathcal{C}_{\mathbb{R}}\left(\left[1, \frac{6}{5}\right]\right)$. Furthermore, since

$$
\mathfrak{L}_{\hat{A}} \mathcal{Q}^{*}+\mathcal{Z}^{*} \simeq 0.9991<1,
$$

Theorem 4 guarantees that only one solution exists for the mentioned mixed multi-order CaputoHadamard-FBVP (16).

Example 2. In the second example, by taking into account the problem (3), we formulated the mixed multi-order Caputo-Hadamard fractional boundary problem:

$$
\left\{\begin{array}{l}
0.99^{\mathcal{C H}} \mathfrak{D}_{1^{+}}^{2.78} u(t)+{ }^{\mathcal{C H}} \mathfrak{D}_{1^{+}}^{2.02} u(t)=\frac{1}{t^{2}+2020}\left(\frac{u(t)}{|u(t)|+1}+\frac{2021}{2022}\right), \\
u(1)=0, \quad 0.01^{\mathcal{C H}} \mathfrak{D}_{1^{+}}^{0.68} u(1.22)+{ }^{\mathcal{C H}} \mathfrak{D}_{1^{+}}^{0.58} u(1.02)=\frac{2022}{2023} \\
0.89^{\mathcal{H}} \mathcal{I}_{1^{+}}^{0.05} u(1.22)+{ }^{\mathcal{H}} \mathcal{I}_{1^{+}}^{02.99} u(1.02)=\frac{2021}{2022}
\end{array}\right.
$$

Here, $t \in[1,1.22], \lambda=0.99, \varsigma=2.78, \theta^{*}=2.02, \gamma_{1}^{*}=0.68, \gamma_{2}^{*}=0.58, q_{1}^{*}=0.05$, $q_{2}^{*}=2.99, \delta_{1}=\frac{2022}{2023}, \delta_{2}=\frac{2021}{2022}, \mu_{1}^{*}=0.01, \mu_{2}^{*}=0.89, \eta=1.02$, and $M=1.22$. Note that: $\gamma_{1}^{*}, \gamma_{2}^{*}<0.76=\varsigma-\theta^{*}$. Based on the above parameters, we can find that:

$$
\begin{aligned}
& \Lambda_{1}^{*} \simeq 0.0227, \quad \Lambda_{2}^{*} \simeq 1.0243, \quad \Lambda_{3}^{*} \simeq 0.0444, \quad \Lambda_{4}^{*} \simeq 0.2233, \\
& \Theta^{*} \simeq 0.0404, \quad \mathcal{Q}^{*} \simeq 0.0130, \quad \mathcal{Z}^{*} \simeq 0.9315, \quad \Delta_{1}^{*} \simeq 2.2112 .
\end{aligned}
$$

In the sequel, regarding $\hat{A}:[1,1.22] \times \mathbb{R} \rightarrow \mathbb{R}$ as the formulation:

$$
\hat{A}(t, u(t))=\frac{1}{t^{2}+2020}\left(\frac{u(t)}{|u(t)|+1}+\frac{2021}{2022}\right) .
$$

Then:

$$
|\hat{A}(t, u(t))|=\left|\frac{1}{t^{2}+2020}\left(\frac{u(t)}{|u(t)|+1}+\frac{2021}{2022}\right)\right| \leq \frac{1}{t^{2}+2020}\left(|u(t)|+\frac{2021}{2022}\right) .
$$

Putting $\Phi_{1}(|u|)=|u|+\frac{2021}{2022}$ and $\Phi_{2}(t)=\frac{1}{t^{2}+2020},|\hat{A}(t, u)| \leq \Phi_{2}(t) \Phi_{1}\left(\|u\|_{\mathcal{W}}\right)$ is valid for any $(t, u) \in[1,1.22] \times \mathbb{R}$. By choosing the constant $\mathfrak{N}^{*}>0$ with $\mathfrak{N}^{*}>32.2674$, we can reach:

$$
\frac{\left(1-\mathcal{Z}^{*}\right) \mathfrak{N}^{*}}{\Phi_{1}\left(\mathfrak{N}^{*}\right)\left\|\Phi_{2}\right\| \mathcal{Q}^{*}+\Delta_{1}^{*}}>1 .
$$

As a consequence, in view of Theorem 5 , we realized that at least one solution function $u$ belonging to $\mathcal{C}_{\mathbb{R}}([1,1.22])$ exists for the mixed multi-order Caputo-Hadamard fractional boundary problem (17).

\section{Conclusions}

In this study, we considered an abstract fractional configuration of the boundary value problem based on the generalized Caputo-Hadamard and Hadamard operators. By defining the Kuratowski measure of noncompactness and recalling its properties, a $\mu$ condensing map was defined. Then, for proving the main existence results, we first applied a fixed point theorem due to Isaia by terms of the topological degree notion, and in the next step, we established the existence criterion by using the Leray-Schauder fixed point theorem. In the last part of the article, we investigated the consistency of our theoretical findings by demonstrating two stimulative examples. 
The work accomplished in this paper is new and enriches the literature on boundary value problems for nonlinear fractional differential equations. For future works, one can extend the given fractional boundary value problem to more general structures, such as finitely point multi-strip integral boundary value conditions given by newly introduced generalized fractional operators with non-singular kernels.

Author Contributions: Conceptualization, S.R., J.T., S.K.N., and S.E.; methodology, S.R., S.B.C., A.A., S.K.N., J.T., and S.E.; formal analysis, S.R., S.B.C., A.A., S.K.N., J.T., and S.E. All authors read and agreed to the published version of the manuscript.

Funding: This research was funded by the Faculty of Applied Science, King Mongkut's University of Technology North Bangkok, Thailand (contract no. 6042101).

Institutional Review Board Statement: Not applicable.

Informed Consent Statement: Not applicable.

Data Availability Statement: Not applicable.

Conflicts of Interest: The authors declare no conflict of interest.

\section{References}

1. Ahmad, B.; Ntouyas, S.K.; Tariboon, J. On hybrid Caputo fractional integro-differential inclusions with nonlocal conditions. J. Nonlinear Sci. Appl. 2016, 9, 4235-4246. [CrossRef]

2. Alsaedi, A.; Baleanu, D.; Etemad, S.; Rezapour, S. On coupled systems of times-fractional differential problems by using a new fractional derivative. J. Funct. Spaces 2016. [CrossRef]

3. Amara, A. Existence results for hybrid fractional differential equations with three-point boundary conditions. AIMS Math. 2020, 5, 1074-1088. [CrossRef]

4. Baleanu, D.; Khan, H.; Jafari, H.; Khan, R.A.; Alipour, M. On existence results for solutions of a coupled system of hybrid boundary value problems with hybrid conditions. Adv. Differ. Equ. 2015, 2015, 318. [CrossRef]

5. Baleanu, D.; Rezapour, S.; Etemad, S.; Alsaedi, A. On a time-fractional integro-differential equation via three-point boundary value conditions. Math. Probl. Eng. 2015, 2015, 12. [CrossRef]

6. Baleanu, D.; Rezapour, S.; Saberpour, Z. On fractional integro-differential inclusions via the extended fractional Caputo-Fabrizio derivation. Bound. Value Probl. 2019, 2019, 79. [CrossRef]

7. Etemad, S.; Ntouyas, S.K. Application of the fixed point theorems on the existence of solutions for $q$-fractional boundary value problems. AIMS Math. 2019, 4, 997-1018. [CrossRef]

8. Etemad, S.; Ntouyas, S.K.; Ahmad, B. Existence theory for a fractional $q$-integro-difference equation with $q$-integral boundary conditions of different orders. Mathematics 2019, 7, 659. [CrossRef]

9. Etemad, S.; Rezapour, S.; Samei, M.E. On fractional hybrid and non-hybrid multi-term integro-differential inclusions with three-point integral hybrid boundary conditions. Adv. Differ. Equ. 2020, 2020, 161. [CrossRef]

10. Mohammadi, H.; Baleanu, D.; Etemad, S.; Rezapour, S. Criteria for existence of solutions for a Liouville-Caputo boundary value problem via generalized Gronwall's inequality. J. Inequl. Appl. 2021, 36, 1-19.

11. Nanware, A.; Dhaigude, D.B. Existence and uniqueness of solutions of differential equations of fractional order with integral boundary conditions. J. Nonlinear Sci. Appl. 2014, 7, 246-254. [CrossRef]

12. Abbas, M.I.; Ragusa, M.A. Solvability of Langevin equations with two Hadamard fractional derivatives via Mittag-Leffler functions. Applicable Anal. 2021. [CrossRef]

13. Aljoudi, S.; Ahmad, B.; Nieto, J.J; Alsaedi, A. A coupled system of Hadamard type sequential fractional differential equations with coupled strip conditions. Chaos Solit. Fract. 2016, 91, 39-46. [CrossRef]

14. Ardjouni, A.; Djoudi, A. Positive solutions for nonlinear Caputo-Hadamard fractional dfferential equations with integral boundary conditions. Open J. Math. Anal. 2019, 3, 62-69. [CrossRef]

15. Derbazi, C.; Hammouche, H. Caputo-Hadamard fractional differential equations with nonlocal fractional integro-differential boundary conditions via topological degree theory. AIMS Math. 2020, 5, 2694-2709. [CrossRef]

16. Tariboon, J.; Cuntavepanit, A.; Ntouyas, S.K.; Nithiarayaphaks, W. Separated boundary value problems of sequential Caputo and Hadamard fractional differential equations. J. Funct. Space. Appl. 2018, 8. [CrossRef]

17. Ntouyas, S.K.; Tariboon, J. Fractional boundary value problems with multiple orders of fractional derivatives and integrals. Electron. J. Differ. Equ. 2017, 2017, 1-18.

18. Lei, X.; Qixang, D.; Gang, L. Existence and Hyers-Ulam stability for three-point boundary value problems with Riemann-Liouville fractional derivatives and integrals. Adv. Differ. Equ. 2018, 2018, 458.

19. Amara, A.; Etemad, S.; Rezapour, S. Topological degree theory and Caputo-Hadamard fractional boundary value problems. Adv. Differ. Equ. 2020, 2020, 369. [CrossRef] 
20. Shah, K.; Ali, A.; Khan, R.A. Degree theory and existence of positive solutions to coupled systems of multi-point boundary value problems. Bound. Value Probl. 2016, 2016, 43. [CrossRef]

21. Shah, K.; Hussain, W. Investigating a class of nonlinear fractional differential equations and its Hyers-Ulam stability by means of topological degree theory. Numer. Funct. Anal. Optim. 2019, 49, 1355-1372. [CrossRef]

22. Zada, M.B.; Shah, K.; Khan, R.A. Existence theory to a coupled system of higher order fractional hybrid differential equations by topological degree theory. Int. J. Appl. Comput. Math. 2018, 4, 102. [CrossRef]

23. Kilbas, A.A.; Srivastava, H.M.; Trujillo, J.J. Theory and Applications of Fractional Differential Equations; Elsevier: Amsterdam, The Netherlands, 2006; Volume 204.

24. Jarad, F.; Baleanu, D.; Abdeljawad, A. Caputo-type modification of the Hadamard fractional derivatives. Adv. Differ. Equ. 2012, 2012, 142. [CrossRef]

25. Agarwal, R.P.; O'Regan, D. Toplogical Degree Theory and Its Applications; Taylor and Francis: Oxfordshire, UK, 2006.

26. Deimling, K. Nonlinear Functional Analysis; Springer: Berlin, Germany, 1985.

27. Banaś, J.; Goebel, K. Measures of Noncompactness in Banach Spaces; Marcel, Dekker Inc.: New York, NY, USA, 1980.

28. Banaś, J.; Olszowy, L. Measures of noncompactness related to monotonicity. Comment. Math. 2001, 41, 13-23

29. Akhmerov, R.R.; Kamenskii, M.I.; Patapov, A.S.; Rodkina, A.E.; Sadovskii, B.N. Measures of Noncompactness and Condensing Operators; Birkhauser: Basel, Switzerland, 1992; Volume 55.

30. Heinz, H.P. On the behaviour of measure of noncompactness with respect to differentiation and integration of vector-valued functions. Nonlinear Anal. 1983, 7, 1351-1371. [CrossRef]

31. Isaia, F. On a nonlinear integral equation without compactness. Acta. Math. Univ. Comen. 2006, 75, $233-240$.

32. Granas, A.; Dugundji, J. Fixed Point Theory; Springer: New York, NY, USA, 2003. 